\title{
An Aerodynamic Analysis of a Spinning Missile with Dithering Canards
}

\author{
Tor A. Nygaard* and Robert L. Meakin ${ }^{\dagger}$ \\ Army/NASA Rotorcraft Division \\ NASA Ames Research Center \\ Moffett Field, CA 94035-1000
}

\begin{abstract}
$\underline{\text { Abstract }}$
A generic spinning missile with dithering canards is used to demonstrate the utility of an overset structured grid approach for simulating the aerodynamics of rolling airframe missile systems. The approach is used to generate a modest aerodynamic database for the generic missile. The database is populated with solutions to the Euler and Navier-Stokes equations. It is used to evaluate grid resolution requirements for accurate prediction of instantaneous missile loads and the relative aerodynamic significance of angle-of-attack, canard pitching sequence, viscous effects, and roll-rate effects. A novel analytical method for inter- and extrapolation of database results is also given.
\end{abstract}

\section{INTRODUCTION}

Simulation of the aerodynamics of rolling airframe missile systems pose significant challenges for any computational approach. Applications of practical interest are characterized by complex vortical flow and shock structures. In addition, the geometry of these missile systems can be very complex, involving relative motion between missile body and control surfaces. The flows are also inherently unsteady. The aim of this paper is to demonstrate the utility of Chimera ${ }^{1}$ overset structured grid domain decomposition methods in the efficient generation of high fidelity aerodynamic simulations for this class of problems.

A generic rolling airframe missile is defined in order to demonstrate the advantages and limitations of an overset grid approach. The paper provides a technical description of the generic missile and the specific computational methods here employed, and complete discussions of the case conditions and corresponding simulation results. The set of simulations considered are designed to demonstrate the level of resolution required for accurate prediction of surface loads and to determine the relative aerodynamic significance of vis- cous and missile roll-rate effects. The aerodynamic performance of the missile as a function of angle-of-attack and canard pitching sequence is also considered. A novel analytical method for describing these performance characteristics is given. A brief summary, acknowledgements, and list of references are provided at the end of the paper.

\section{ROLLING AIRFRAME CONFIGURATION}

The generic rolling airframe missile employed in the present work is referred to as FM-3. The FM-3 missile has a hemispherical nose, cylindrical body, four fins, and two canards. Details of the geometric complexity of the missile are illustrated in Figure 1.

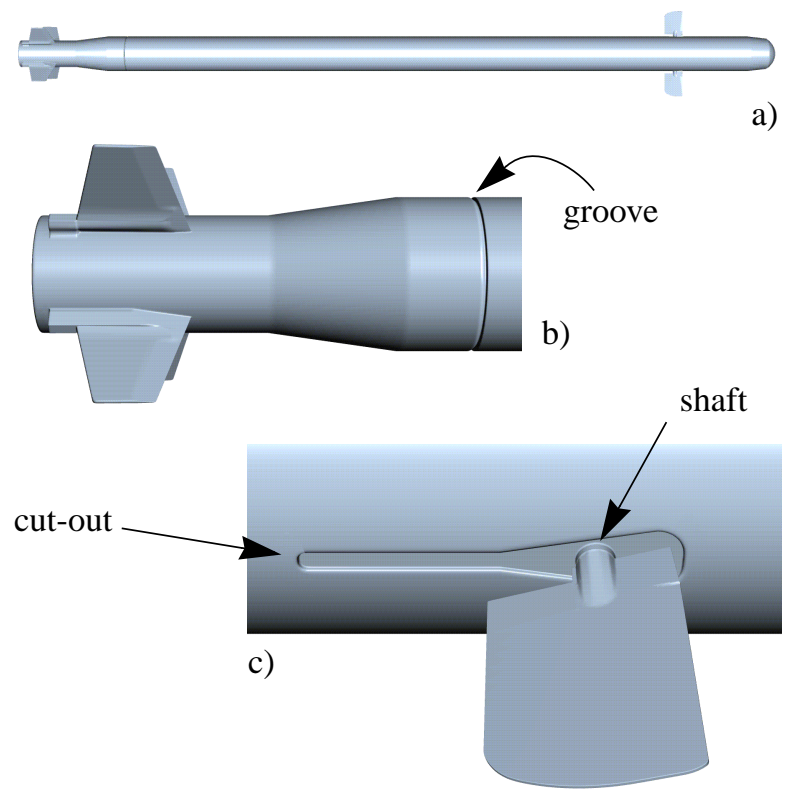

Figure 1. FM-3 missile geometry. a) Top view of entire missile. b) Fins. c) Close-up of canard.

\footnotetext{
* Research Scientist, ELORET Corp., Member AIAA

${ }^{\dagger}$ Senior Research Scientist, U.S. Army AFDD (AMCOM), Senior Member AIAA

This material is declared a work of the U.S. Government and is not subject to copyright protection in the United States.
} 
The fins are designed to induce missile spin, while directional control is actuated via canard dithering. As the missile spins, the canard pitch position follows an actuator signal with constant pitch rate. The actuator signal flip-flops between +/- 1 according to the sign of the sum of two sine-waves called the command and dither signals. The amplitude of the command signal relative to the dither signal is called the command level, and reflects the strength of the attempted maneuver. The command signal is modulated with the roll-rate. The dither signal is modulated with a dither frequency. Figure 2 shows the canard pitching algorithm for a command level of $100 \%$ for the specified roll-rate and dither frequency.

\section{$\underline{\text { 3. COMPUTATIONAL METHODS }}$}

\subsection{Discretization Method}

The "near-body" and "off-body" domain partitioning method described in References 2 and 3 is used here as the basis of discretization of the FM-3 missile. In the approach, the near-body portion of a domain is defined to include the surface geometry of all bodies being considered and the volume of space extending a short dis- tance away from the respective surfaces. The construction of near-body grids and associated intergrid connectivity is a classical Chimera-style decomposition of the near-body domain. It is assumed that near-body grids provide grid point distributions of sufficient density to accurately resolve the flow physics of interest (i.e., boundary-layers, vortices, etc.) without the need for refinement. This is a reasonable constraint since near-body grids are only required to extend a short distance away from body surfaces. Figure 3 illustrates the surface decomposition of the FM-3 missile and shows selected surfaces from the resulting near-body surface and volume grids.

The off-body portion of the domain is defined to encompass the near-body domain and extend out to the far-field boundaries of the problem. The off-body domain is filled with overlapping uniform Cartesian grids of variable levels of refinement, as shown in Figure 4 for the FM-3 missile. The off-body grid resolution amplification factor between successive levels is 2 . The near-body off-body partitioning approach facilitates grid adaptation in response to proximity of body components and/or to estimates of solution error within the topologically simple off-body grid system.

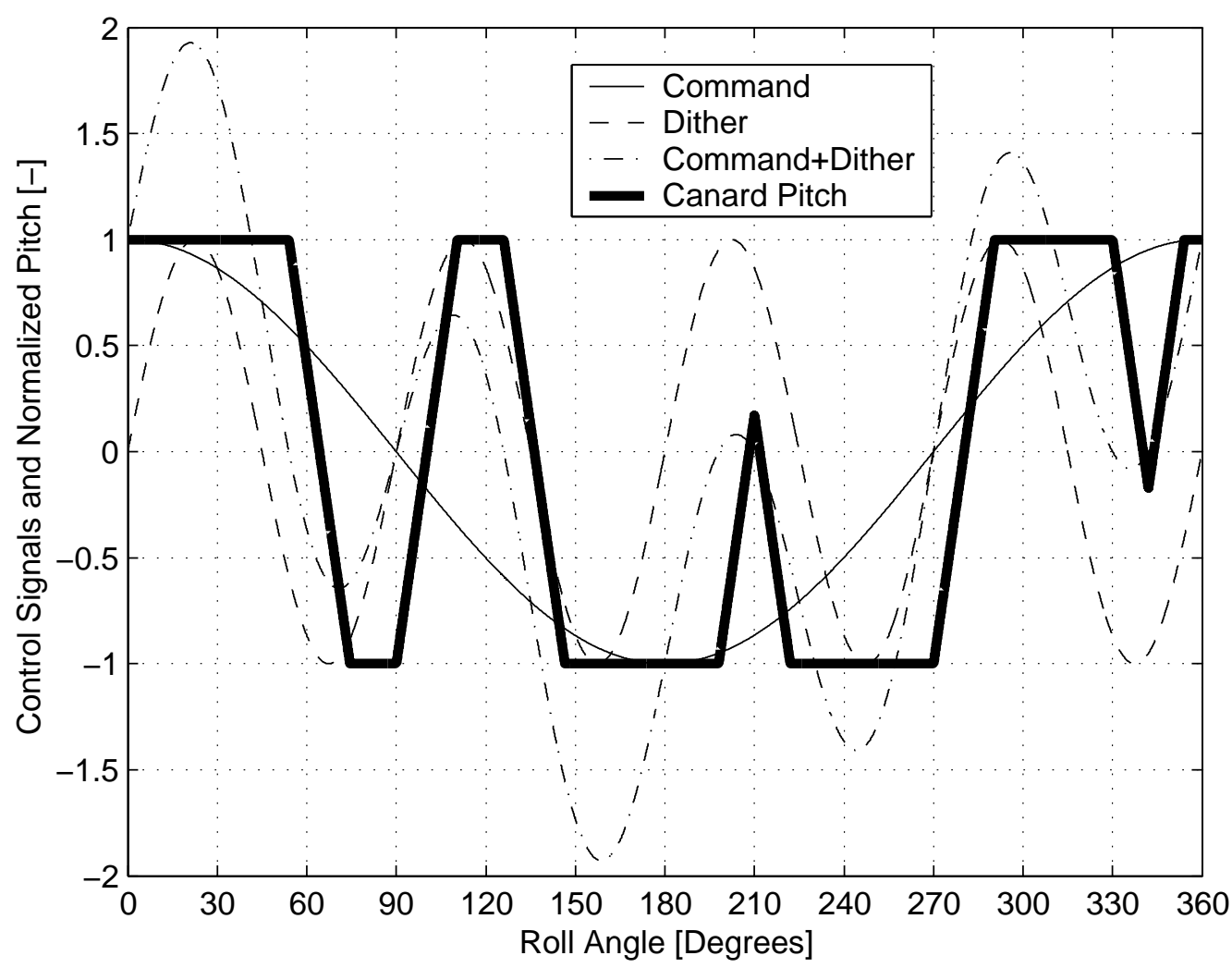

Figure 2. Canard pitching algorithm for roll-rate of $8.75 \mathrm{~Hz}$, dither frequency of $35 \mathrm{~Hz}$, and $100 \%$ command level. 


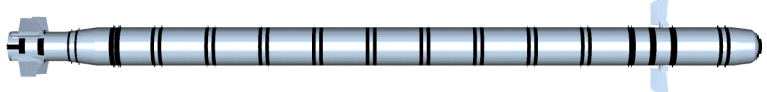

a)

b)
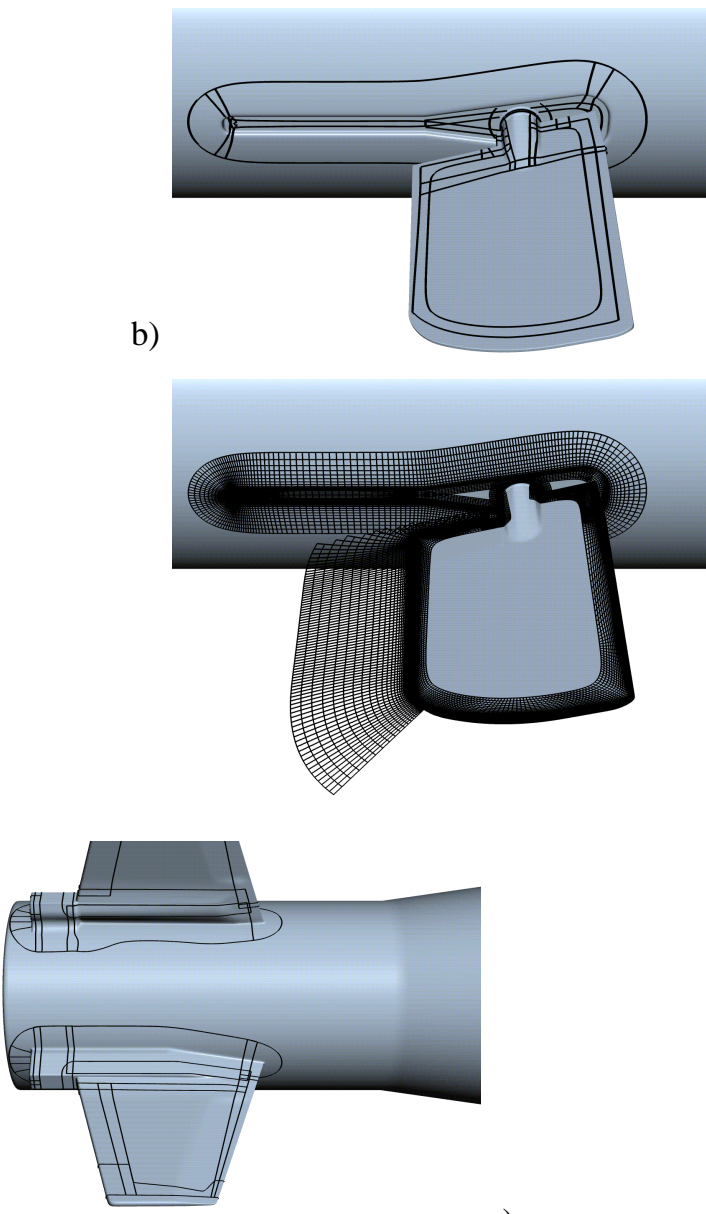

c)

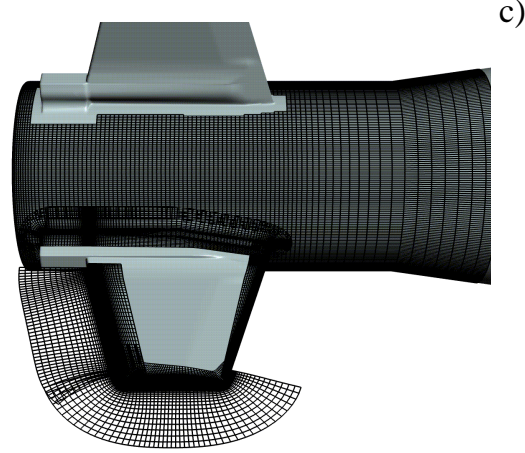

Figure 3. FM-3 surface geometry decomposition and near-body grids. a) Surface decomposition of missile body. b) Decomposition of canard surfaces and selected surfaces from corresponding grids. c) Decomposition of fin surfaces and selected surfaces from corresponding grids.

Note: Surface geometry decomposition and near-body grid generation accomplished using OVERGRID utility from $\mathrm{CGT}^{4}$.

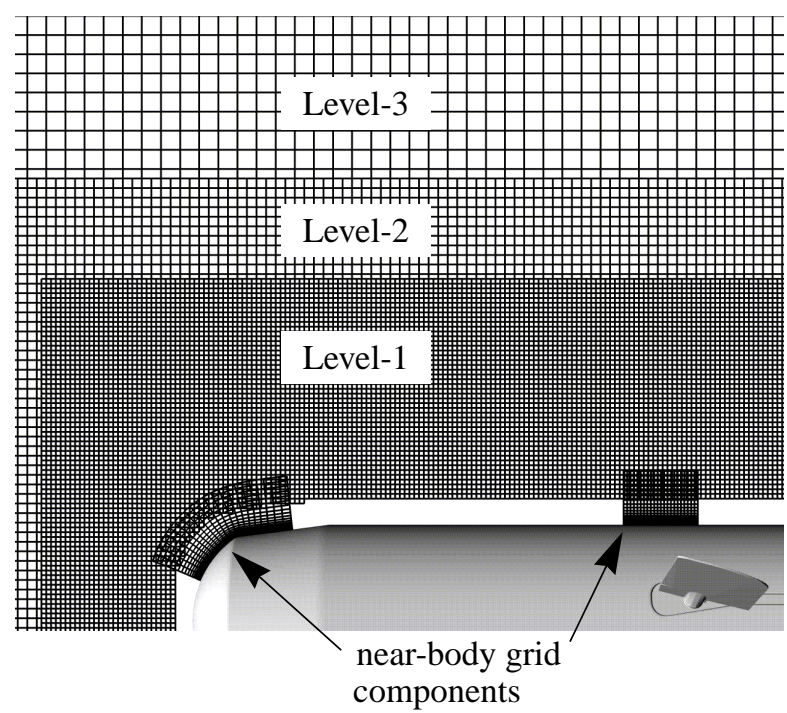

Figure 4. FM-3 near-body and off-body partitioning and selected surfaces.

\subsection{Solution Method}

The set of FM-3 missile simulations presented in this paper represent a wide variety of conditions and are products of the OVERFLOW- $\mathrm{D}^{3,5}$ code. OVERFLOW$\mathrm{D}$ is based on version 1.6au of the well known NASA OVERFLOW $^{6}$ code, but has been significantly enhanced to accommodate moving body applications. The OVERFLOW-D enhancements represent in-core subroutine actuated operations and include the following capabilities.

$i$. On-the-fly generation of off-body grid systems.

ii. $\mathrm{MPI}^{6}$ enabled scalable parallel computing.

iii. Automatic load balancing.

iv. Aerodynamic force and moment computations.

v. General 6-degrees-of-freedom model.

$v i$. Rigid-body relative motion between an arbitrary number of bodies.

vii. Domain connectivity.

viii. Solution error estimation.

ix. Grid adaptation in response to body motion and/or estimates of solution error.

The majority of the FM-3 simulations presented in this paper involve relative motion between grid components. The entire missile spins relative to the inertial off-body grid system and the canards dither relative to the missile body. The pseudo-code below outlines the general procedure used in OVERFLOW-D to carry out such simulations. Of course, the flow equations are solved at every time-step during a simulation. In cases that involve relative motion between configuration components, body dynamics and domain connectivity are 
also addressed at each time-step. In the case of the FM3 missile, body dynamics simply means the computation of aerodynamic loads and moving the missile components according to a control-law. Specifically, the rotational orientation of the missile is positioned as a function of time and the roll-rate. The position of the canards relative to the missile body is determined by the canard dither algorithm (illustrated in Figure 2).

\section{For $\mathrm{N}$ time-steps}

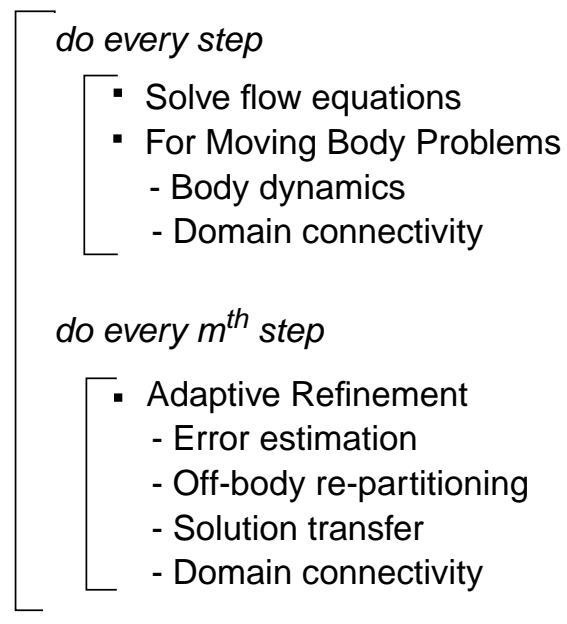

Pseudo-Code. Solution procedure (with adaptive refinement capability) for unsteady problems that may involve relative motion between component parts.

Since the missile movement is continuous, the relative position of many grid components change every time-step. In order for solution information to be correctly exchanged between grids during the simulation, the domain connectivity solution must also be continuously updated. This is accomplished automatically by OVERFLOW-D.

The OVERFLOW-D processing rate for static geometry viscous flow applications is about $15 \mu \mathrm{sec}$ per grid-point per time-step (300MHz processor). For moving-body problems, the processing rate is somewhat problem dependent, but generally falls in the bounds of 15 to $18 \mu$ sec per grid-point per time-step. For the FM3 spinning missile cases considered here, the number is $16.5 \mu \mathrm{sec}$ per grid-point per time-step. OVERFLOW-D accommodates problem sizes of more than 2 million grid-points per 1 gigabyte of memory. Maximum parallel efficiency (percentage in high 90's) is realized when the fewest number of processors that can accommodate a given problem in core memory are selected. OVERFLOW-D can efficiently (i.e., over 70\%) make use of larger numbers of processors for a fixed problem size when each processor assumes the load of at least 250 thousand points. Load balancing is an automatic function of OVERFLOW-D.

As indicated in the pseudo-code above, OVERFLOW-D accommodates solution adaptation based on the position of near-body grid components and/or in response to estimates of solution error. The off-body grid management scheme allocates level-1 (finest) resolution grids to accommodate significant motion of body components or flow features before the next adapt cycle. Accordingly, adapt cycles are only required periodically; every 25 to 50 time-steps in a typical unsteady simulation.

In the FM-3 missile cases considered here, all flow features that are likely to have any significant affect on the surface forces and moments are confined to the volume of space within a missile diameter of the body itself. These include canard vortices, boundary layer, and key portions of the shock systems. Accordingly, OVERFLOW-D input is used to allocate level-1 resolution capacity to a distance of 1.5 diameters from the missile surface, rather than enable adaptation in response to solution error. A slight savings in computational overhead is thereby gained for the present cases.

\section{SIMULATION RESULTS}

A set of FM-3 missile simulations is carried out to demonstrate the level of resolution required for accurate prediction of surface forces and moments and to determine the relative aerodynamic significance of viscous effects, missile roll-rates $\left(\Omega_{\mathrm{r}}\right)$, canard command levels $(c)$, and free-stream angles-of-attack $(\alpha)$. A total of 31 FM-3 simulations form the basis of the material presented in this paper concerning these issues. The flight conditions for the cases are indicated in Table 1. The parameters varied to obtain the complete simulation set are free-stream angle-of-attack, canard pitch command level, and missile roll-rate. The other simulation parameters indicated in the table are held fixed and are common to all cases considered.

Table 1. Simulation Parameters

\begin{tabular}{|l|l|l|}
\hline $\mathrm{M}_{\infty}$ & Mach number & 1.6 \\
$\mathbf{R e}$ & Reynolds number & $50 \times 10^{6}$ \\
$\Omega_{\mathrm{d}}$ & Dither-frequency & $35 \mathrm{~Hz}$ \\
\hline$\alpha$ & Angle-of-attack & $0^{\mathrm{O}}, 2^{\mathrm{O}}, 3^{\mathrm{O}}, 4^{\mathrm{O}}, 8^{\mathrm{O}}, 12^{\mathrm{O}}, 15^{\mathrm{O}}$ \\
$\boldsymbol{c}$ & Command level & $0 \%, 100 \%, 200 \%$ \\
$\Omega_{\mathrm{r}}$ & Roll-rate & $0 \mathrm{~Hz}, 8.75 \mathrm{~Hz}$ \\
\hline
\end{tabular}


The general characteristics of the FM-3 flow field are illustrated in Figure 5. Vortices are shed from the inboard and outboard tips of the canard and convect down the length of the missile interacting with the viscous boundary layer. Away from the influence of the boundary layer, the outboard canard vortices twist approximately $8^{\circ}$ around the spinning missile in one body-length of travel. As can be seen in the figure, disruptions to the boundary layer by the inboard canard vortices are dragged through nearly $45^{\circ}$ of roll over the same interval. The shock structure is indicated in Figure $5 \mathrm{~b}$. The boundary layer growth on the upper surface of the missile is also visible in the figure. A positive angleof-attack and vortex/boundary layer interaction combine to exaggerate the boundary layer thickness down the stream-wise axis of the missile.
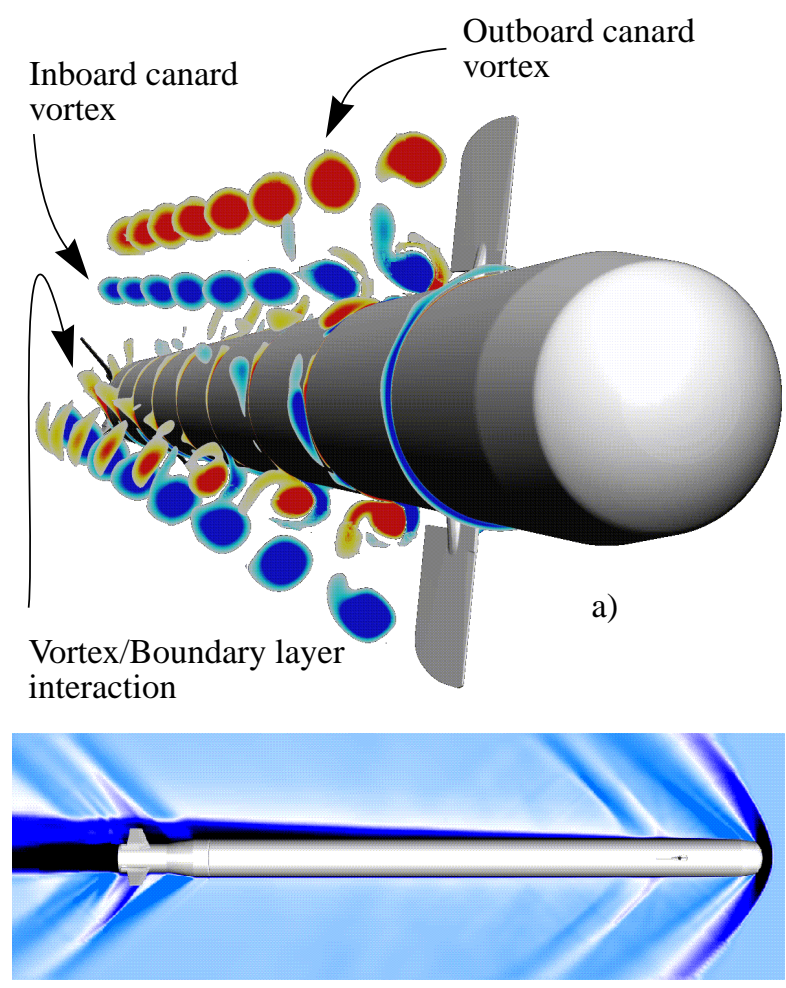

b)

Figure 5. Aerodynamics of the FM-3 spinning missile with dithering canards. a) Vortex structure. b) Shock structure. $\Omega_{\mathrm{r}}=8.75 \mathrm{~Hz}, \boldsymbol{c}=0 \%, \alpha=3^{\circ}$.

\subsection{Resolution Requirements}

A grid refinement study is used to determine the level of spatial resolution needed to accurately predict the integrated FM-3 surface loads. The significance of grid resolution is evaluated here by comparing viscous solutions for the spinning missile case defined in Table 1 with the variable parameters $c$, $\alpha$, and $\Omega_{\mathrm{r}}$ fixed at $0 \%$, $3^{\circ}$, and $8.75 \mathrm{~Hz}$, respectively. A very high resolution grid is used to define the baseline solution. Medium and coarse solutions are obtained on grids derived from the baseline grid with successively lower levels of spatial resolution. The qualitative effect of coarsening on the surface geometry is shown in Figure 6. A very high fidelity temporal resolution (viz., 12,000 time-steps per missile revolution) is uniformly employed in all of the viscous simulations.
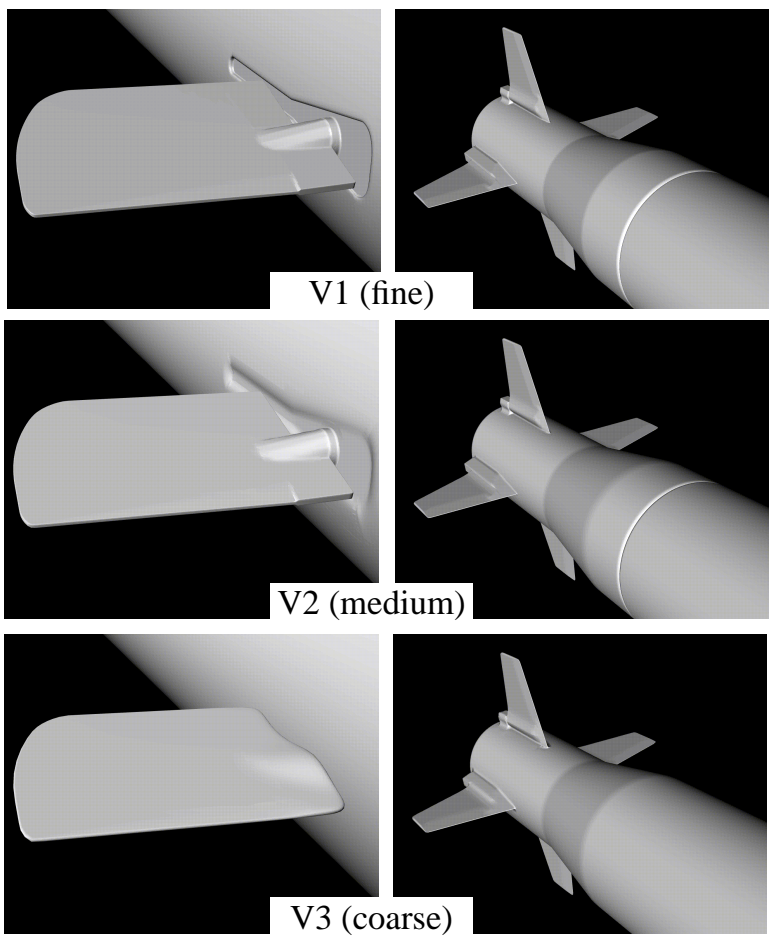

Figure 6. Fine, medium, and coarse grid representations of FM-3 canards and fins.

The baseline grid for this case (finest resolution) is comprised of 41 million grid points and is referred to hereafter as the V1 (i.e., "Viscous-1") grid. Isolated surfaces from the V1 grid are shown in Figures 3 and 4. The flow characteristics illustrated in Figure 5 are from a simulation using the V1 grid. All grid lengths referred to in the following discussion are normalized by the missile body length. Viscous spacing normal to the body surfaces is $2.5 \times 10^{-6}$ in the V1 grid. This corresponds to a $\mathrm{y}^{+}$of 1 for a Reynolds number of 10 million. This spacing is maintained uniformly across the first 6 cells in the viscous direction and then expanded with a geometric stretching ratio of 1.2 to a distance of approximately 0.015 . The maximum spacing used in the nearbody grids is approximately equal to the level-1 offbody grid spacing which is 0.0013 , or approximately $0.1 \%$ of the body length. 
The V1 grid is the basis of the medium (V2) and coarse (V3) grids. The V2 grid is obtained by deleting approximately every other point from the $\mathrm{V} 1$ grid in all three spatial dimensions and results in a grid with just over 8 million points. Similarly, the V3 grid is obtained from the V2 grid by deleting approximately every other point from the V2 grid in all three spatial dimensions and results in a grid with just over 2 million points. The foregoing is true subject to the following qualifications.

$i$. Some surface grids require redistribution and/ or addition of grid-points to preserve geometric features such as sharp corners through the two subsequent eliminations of every other point.

ii. Smoothing is applied to geometric features that are not adequately resolved by the coarser grids. For the V2 grid, smoothing is applied to the canard cut-out and the missile groove. For the V3 grid, the canard cut-out, the canard shaft and the missile groove are removed completely.

iii. The grid spacing in the surface normal direction for the V2 grid corresponds approximately to every other point for the V1 grid, doubling the initial spacing from the wall.

$i v$. The grid spacing in the surface normal direction for the V3 grid starts at the surface with the V2 spacing doubled. The stretching ratio thereafter is approximately the same as in the V2 grid.

Consider now the computed load histories obtained from simulations using the V1, V2, and V3 grids. Figure 7 shows the computed normal force $(\mathrm{Cz})$ history for the three different resolution capacities. The canard pitch angle history is also indicated. The V1 and V2 results are in good agreement, except at maximum canard deflection. At high canard lift, the strong vortices shed from the canards modify the pressure distribution on the fuselage and the tail fins. Still, the rollaveraged normal forces from V1 and V2 shown in Table 2 differ by less than $0.3 \%$, indicating near grid convergence for this quantity. The V3 result differs significantly from V1 and V2.

The data represented in Figures 8 and 9 observe the same form as that used in Figure 7. However, Figures 8 and 9 display side force $(\mathrm{Cy})$ and axial force $(\mathrm{Cx})$ histories, respectively. The side forces exhibit the same effect as for the normal forces at maximum canard deflection. The roll-averaged side-forces are close to zero, with a difference between V1 and V2 of less than $0.02 \%$ of the normal force, indicating grid convergence for this quantity also. In contrast, the axial forces (see
Table 2. Roll-averaged force and moment coefs. *

\begin{tabular}{|l|c|l|c|}
\hline Coefficient & Fine (V1) & Med. (V2) & Coarse (V3) \\
\hline Cx (axial) & 1.17 & 1.12 & 1.07 \\
Cy (side) & $-7.56 \mathrm{e}-03$ & $2.76 \mathrm{e}-03$ & $-1.21 \mathrm{e}-03$ \\
Cz (normal) & 0.461 & 0.462 & 0.538 \\
Cmx (roll) & $-1.19 \mathrm{e}-03$ & $-1.08 \mathrm{e}-03$ & $-0.94 \mathrm{e}-03$ \\
Cmy (pitch) & $-6.79 \mathrm{e}-03$ & $-3.11 \mathrm{e}-03$ & $-3.45 \mathrm{e}-02$ \\
Cmz (yaw) & $2.80 \mathrm{e}-03$ & $3.52 \mathrm{e}-03$ & $-9.55 \mathrm{e}-04$ \\
\hline
\end{tabular}

*Moments are about the missile center of gravity

Figure 9), indicate a systematic shift higher for finer resolution. The difference between V1 and V2 axial forces is approximately the same as between the corresponding V2 and V3 results. Clearly, grid convergence is not apparent in the computed axial force data.

Figures 10 and 11 break down the axial forces into pressure and viscous components. The V2 and V3 solutions are almost identical for the pressure component; and V1 has a systematic shift to a higher value. The contribution to this shift comes mainly from the aft part of the missile. This region of the flow has complicated interactions between the expansion waves around the boat-tail, shocks around the tail fins, and the boundary layer. The V1 and V2 viscous components to the axial force differ by approximately $2 \%$ of the total axial force, slightly less than the difference between the V2 and V3 results. The total roll-averaged axial forces are shown in Table 2. The medium and coarse solution axial forces are $4 \%$ and $8 \%$ lower than for the corresponding fine solution. The reason grid convergence in axial force is not demonstrable via the current set of solutions is not clear. It may be that while the $\mathrm{V} 1$ grid has sufficient resolution in the boundary layer, the V2 and V3 viscous spacing (double and quadruple that of V1) is not sufficient.

Figure 12 shows the pitching moment (Cmy) about the center of gravity for the V1, V2, and V3 simulations. The missile center of gravity is located approximately at the missile midpoint. As is the case for normal force, the differences in pitching moment are largest at maximum canard deflection. Still, the overall agreement is very good. The difference between the V1 and V2 rollaveraged pitching moments are approximately $1 \%$ of the maximum pitching moment during a revolution. The roll-averaged pitching moments are shown in Table 2. The percentage of maximum pitching moment is used here as a measure of grid convergence since the rollaveraged moments are all nearly zero.

Figure 13 shows the yawing moment (Cmz) about the center of gravity. The agreement is very good, with 


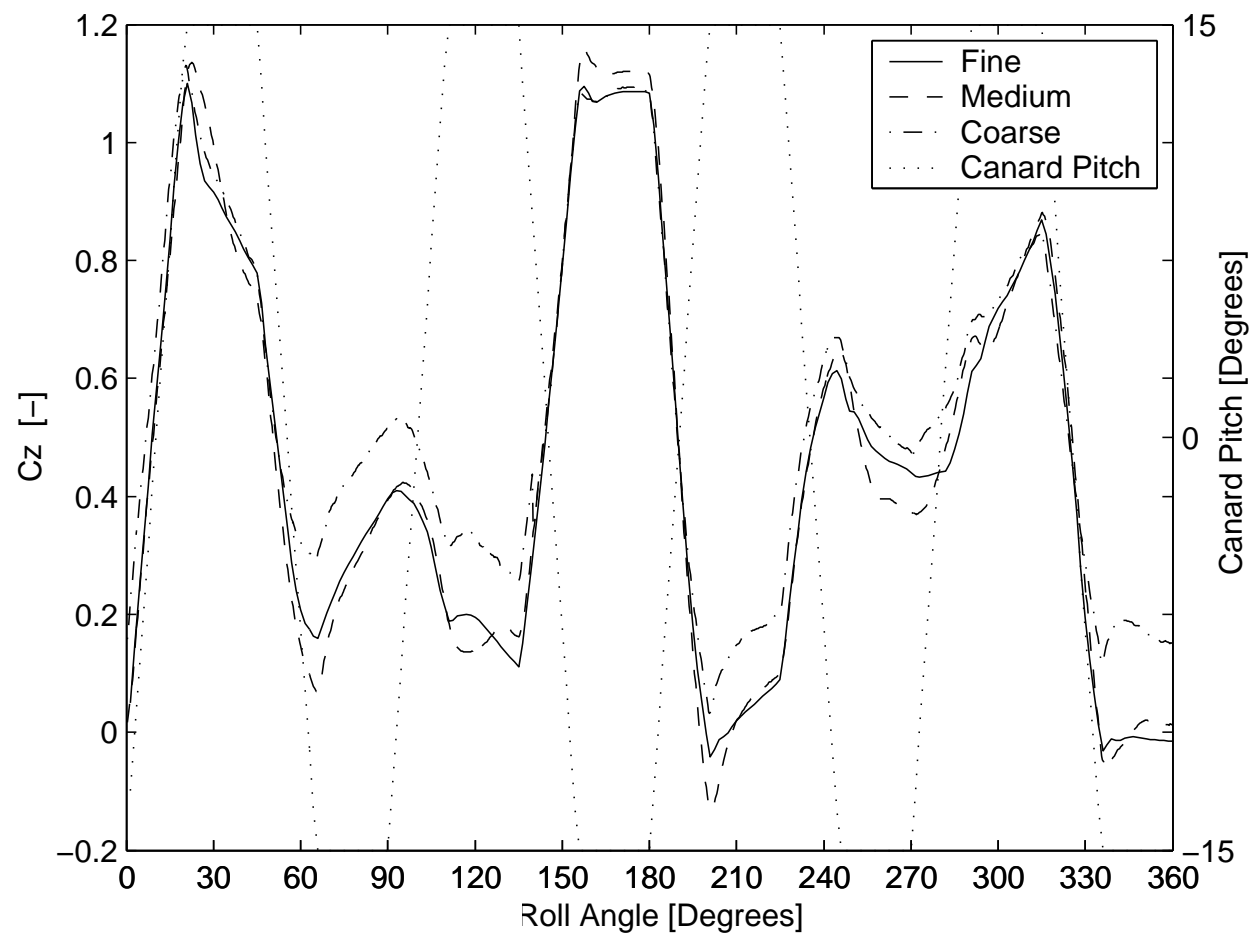

Figure 7. Grid effects in the NORMAL FORCE coefficient.

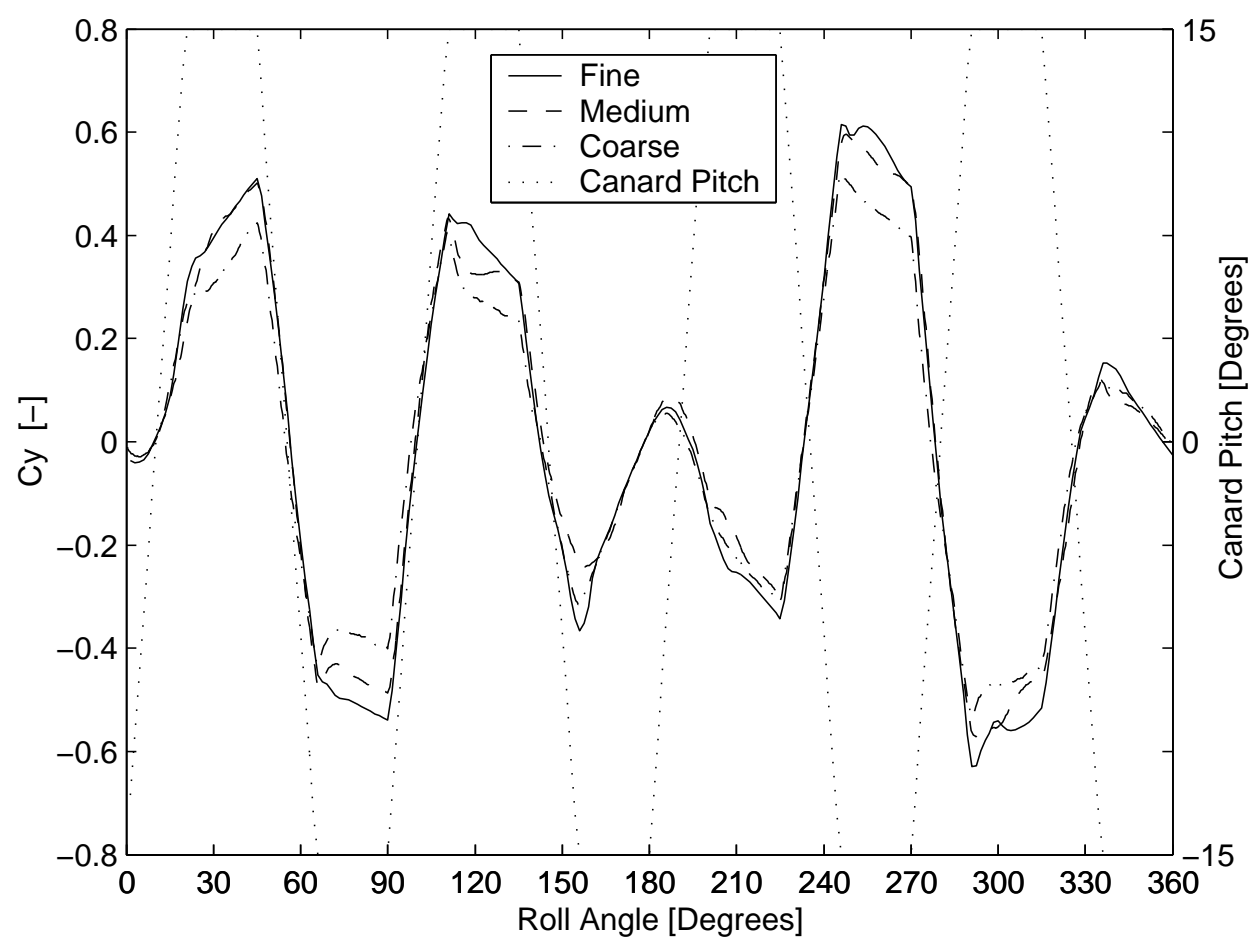

Figure 8. Grid effects in the SIDE FORCE coefficient. 


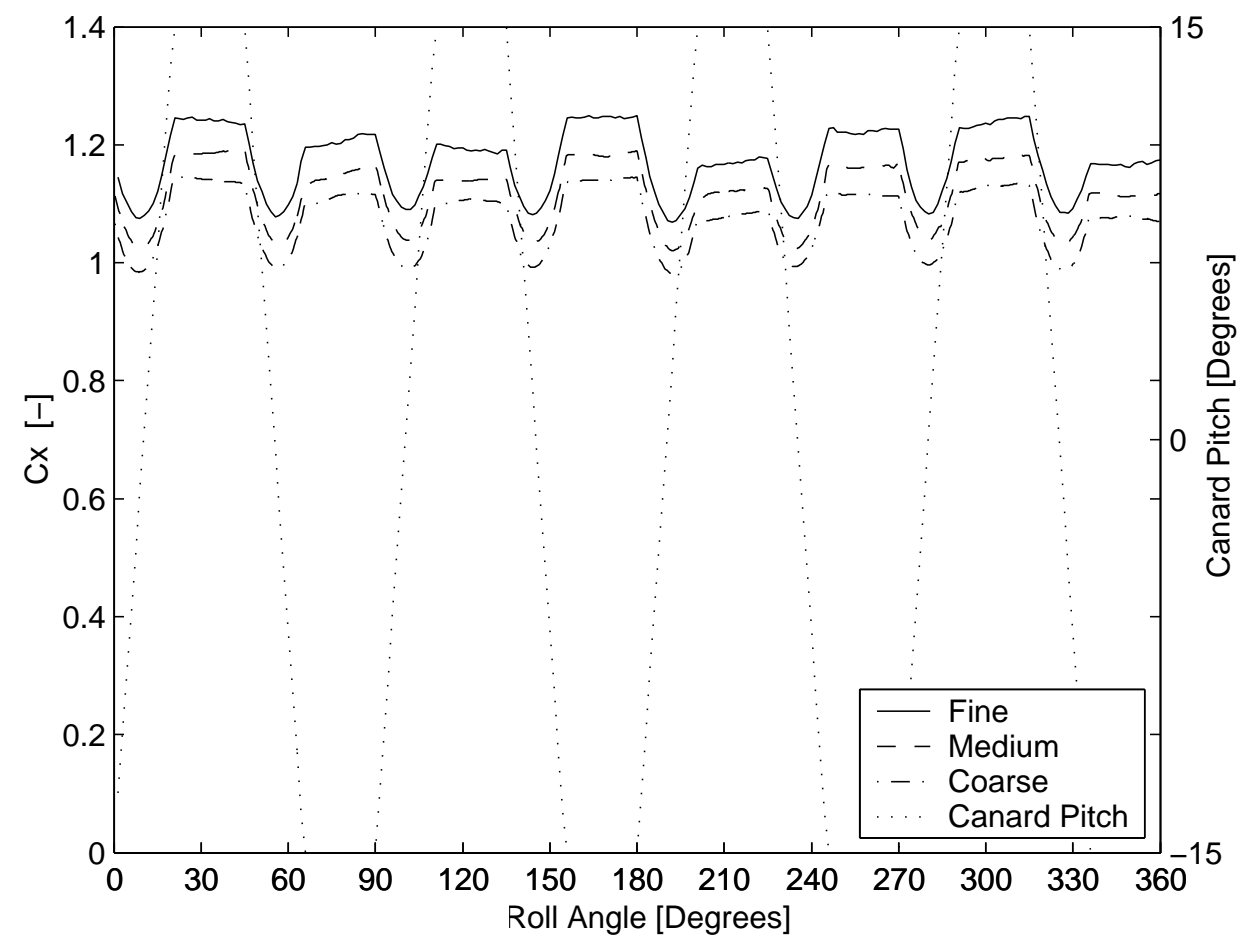

Figure 9. Grid effects in the AXIAL FORCE coefficient.

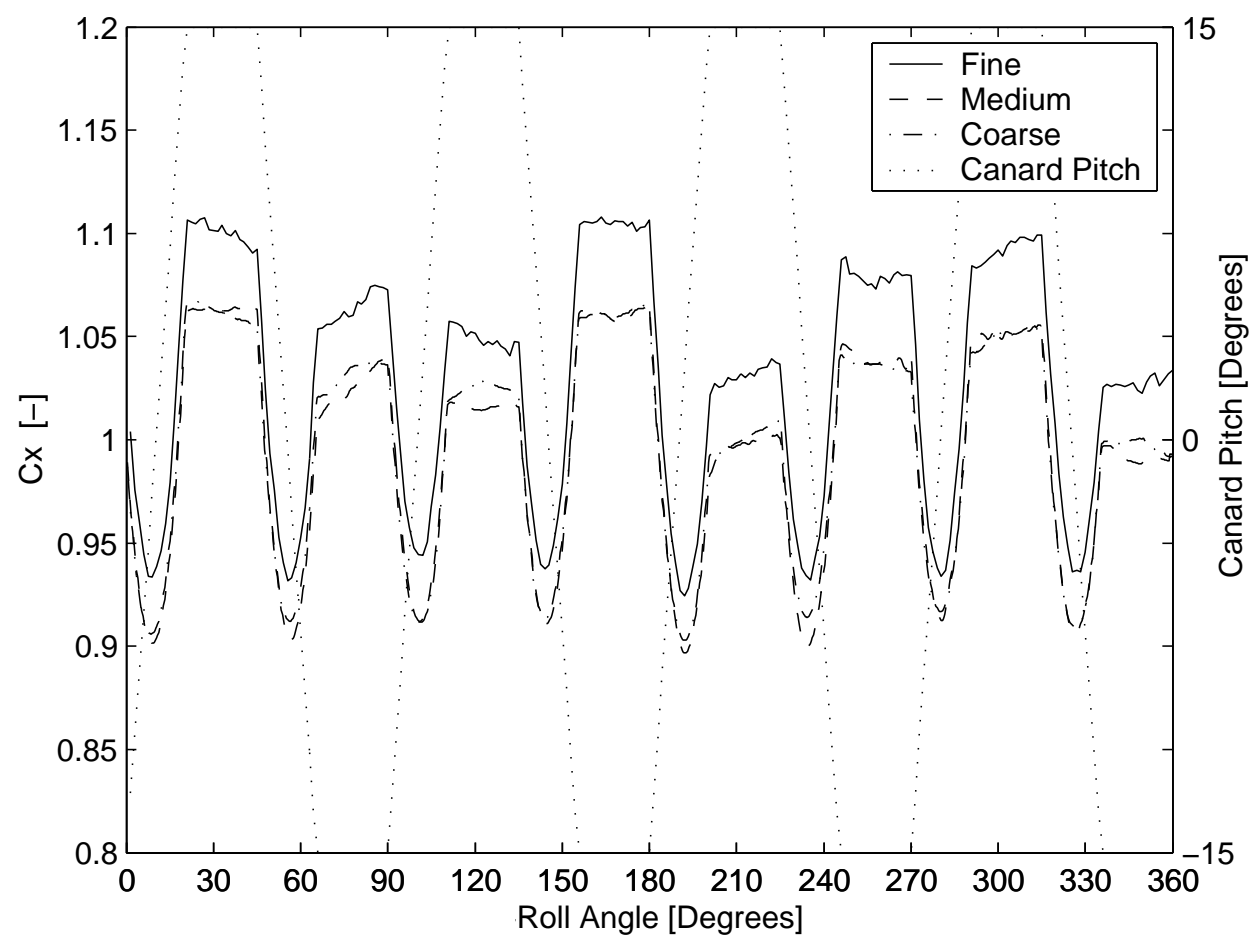

Figure 10. Grid effects in the AXIAL FORCE coefficient (PRESSURE component). 


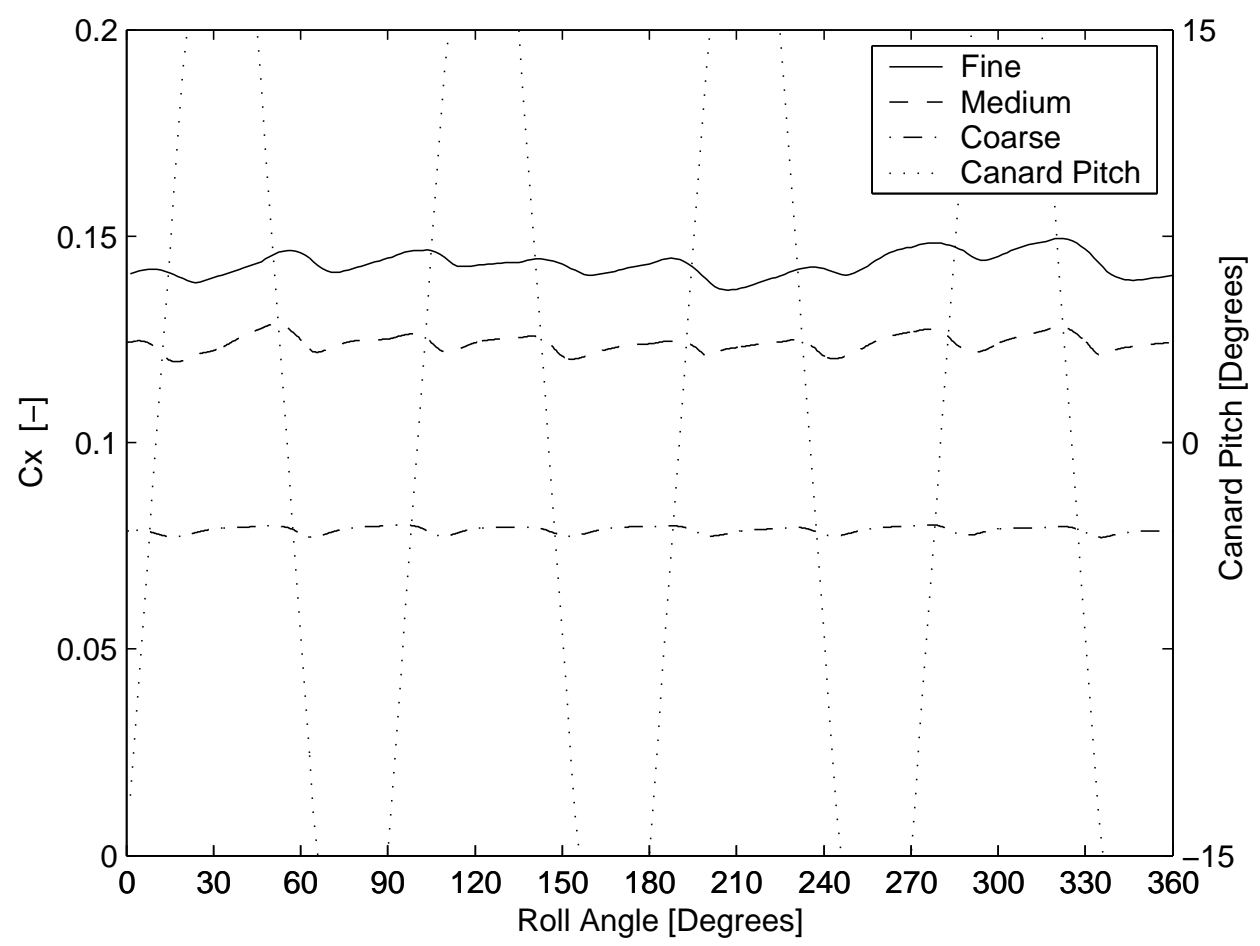

Figure 11. Grid effects in the AXIAL FORCE coefficient (VISCOUS component).

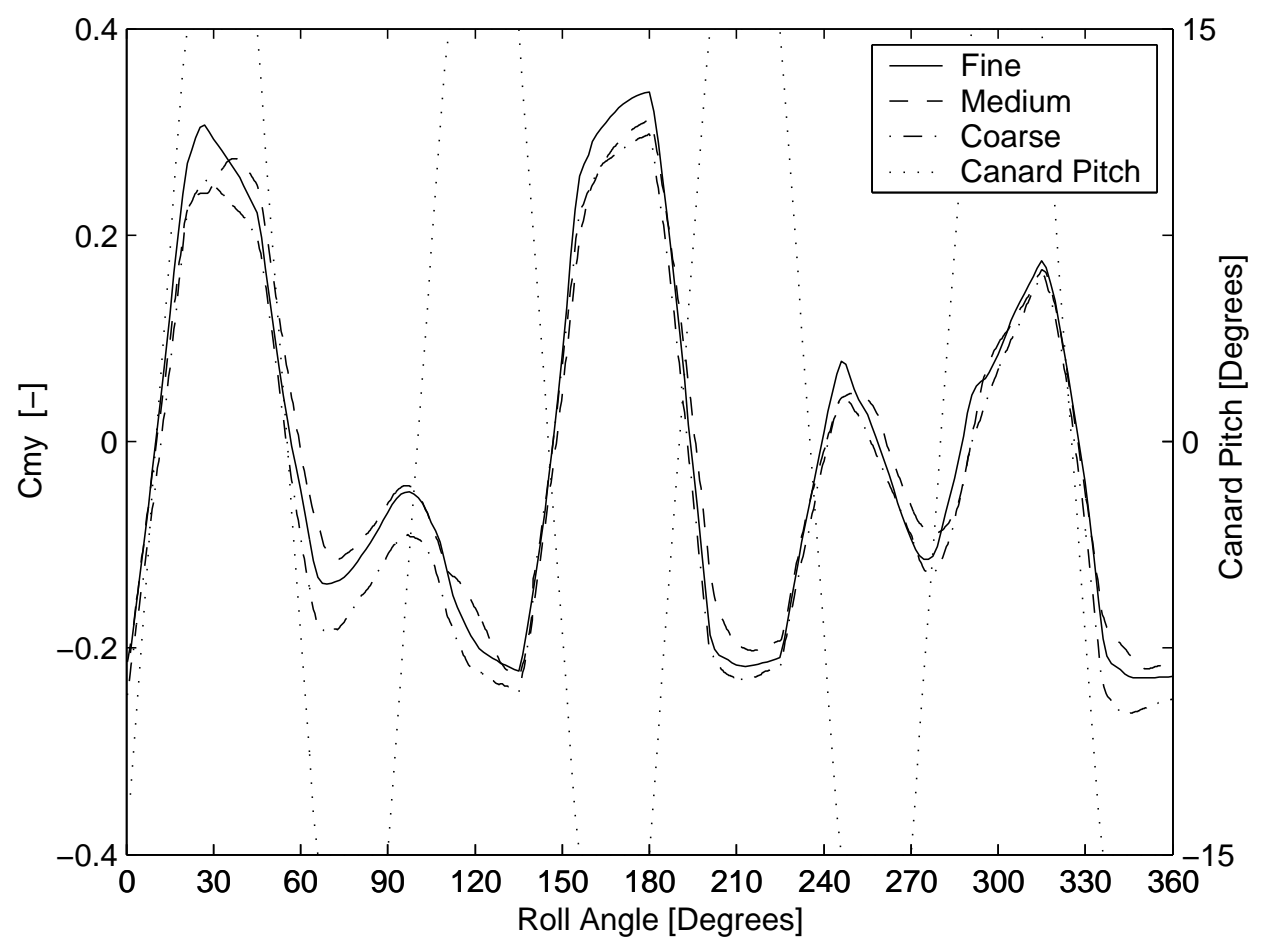

Figure 12. Grid effects in the PITCHING MOMENT coefficient. 


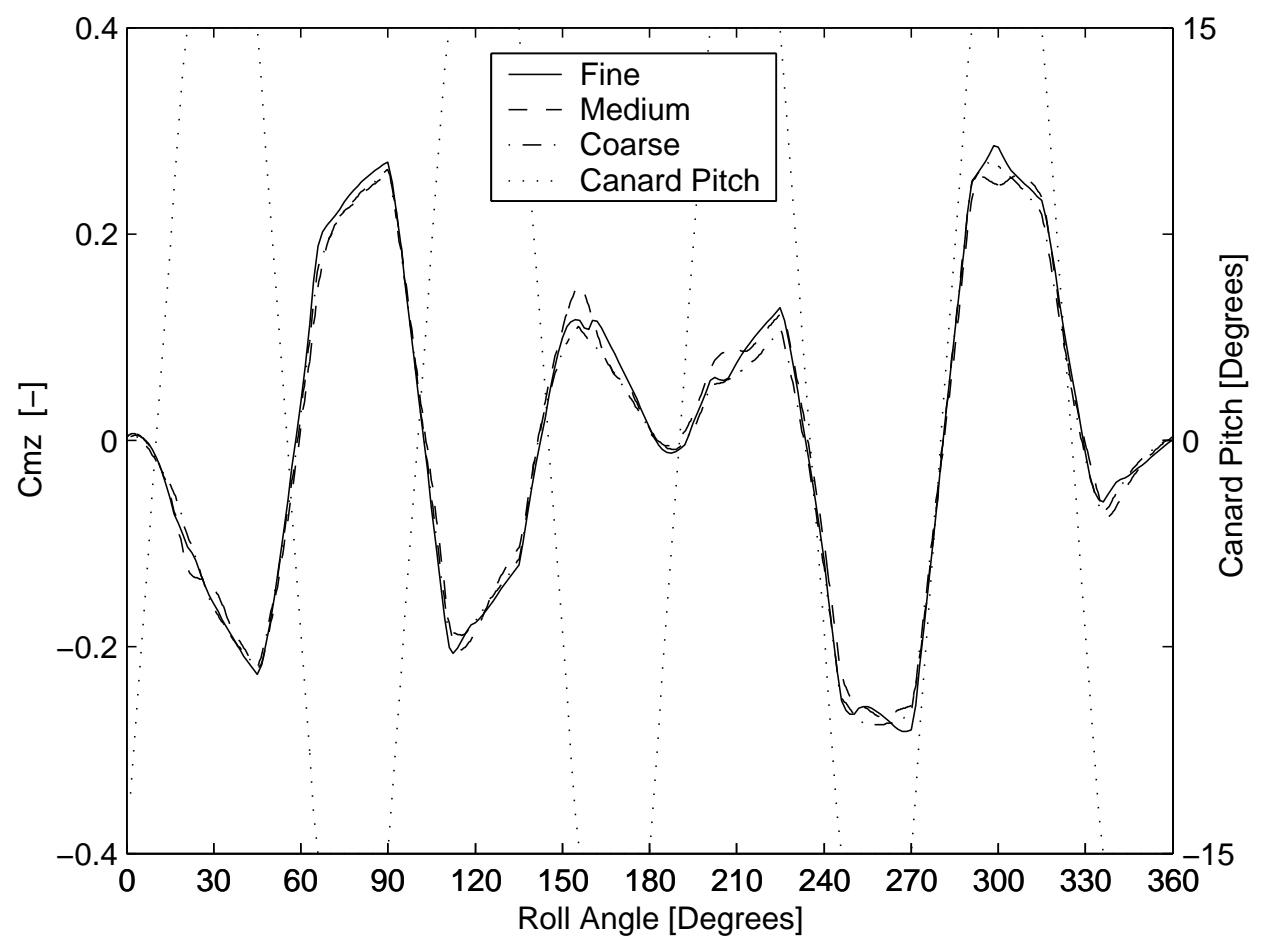

Figure 13. Grid effects in the YAWING MOMENT coefficient.

some minor differences at maximum canard deflection. The difference between the V1 and V2 roll-averaged yawing moments are approximately $0.2 \%$ of the maximum pitching moment during a revolution. The rollaveraged yawing moments are shown in Table 2 .

The rolling moment $(\mathrm{Cmx})$ is shown in Figure 14. As expected, the moments are small. The difference between the V1 and V2 roll-averaged rolling moments are approximately $0.03 \%$ of the maximum pitching moment during a revolution. A steady-state freely spinning missile should have an averaged rolling moment of zero. The overall pattern here is in fair agreement, but the details differ significantly. The rolling moment is sensitive to flow details around the tail fins, with complex interactions between the boundary layer, the canard vortices and the shocks, which again depend on the accurate prediction of the flow along the entire missile. Grid convergence for so small a quantity as the rolling moment is beyond the capacity of the present set of grids.

The effect of grid resolution on the prediction of the aerodynamic details of the flow is illustrated in Figure 15. Figures $15 \mathrm{a}, 15 \mathrm{~b}$, and $15 \mathrm{c}$ provide a comparative view of the canard vortices and missile boundary layer interaction via plots of helicity density (i.e., dot product of the velocity and vorticity vectors) at several stations along the length of the missile (for a roll-angle of $268.5^{\circ}$ at maximum negative canard deflection). The position of the vortices are in good agreement for the V1 and V2 solutions. However, the vortex strength is weaker and interactions between the inboard canard vortex and viscous boundary layer of the missile body are less apparent in the V2 solution. The V3 solution differs significantly from the V1 and V2 solutions in vortex position, strength, and vortex/boundary layer interaction.

The grid refinement results suggest very good overall agreement between the V1 and V2 solutions, though some differences are apparent. Still, the V2 grid offers a good compromise between solution accuracy and solution throughput for computations designed to predict aerodynamic forces and moments. As noted above, grid convergence of all forces and moments (except axial force and rolling moment) are obtained.

\subsection{Viscous Effects}

A comparative evaluation of very high resolution Navier-Stokes and Euler simulations is used to determine the relative significance of viscous effects operative in the range of flight conditions considered for the FM-3 missile. The case conditions defined in Table 1 are taken as representative of these flight conditions. A static FM-3 case is first considered where the missile roll-rate is zero and the canards are fixed in neutral posi- 


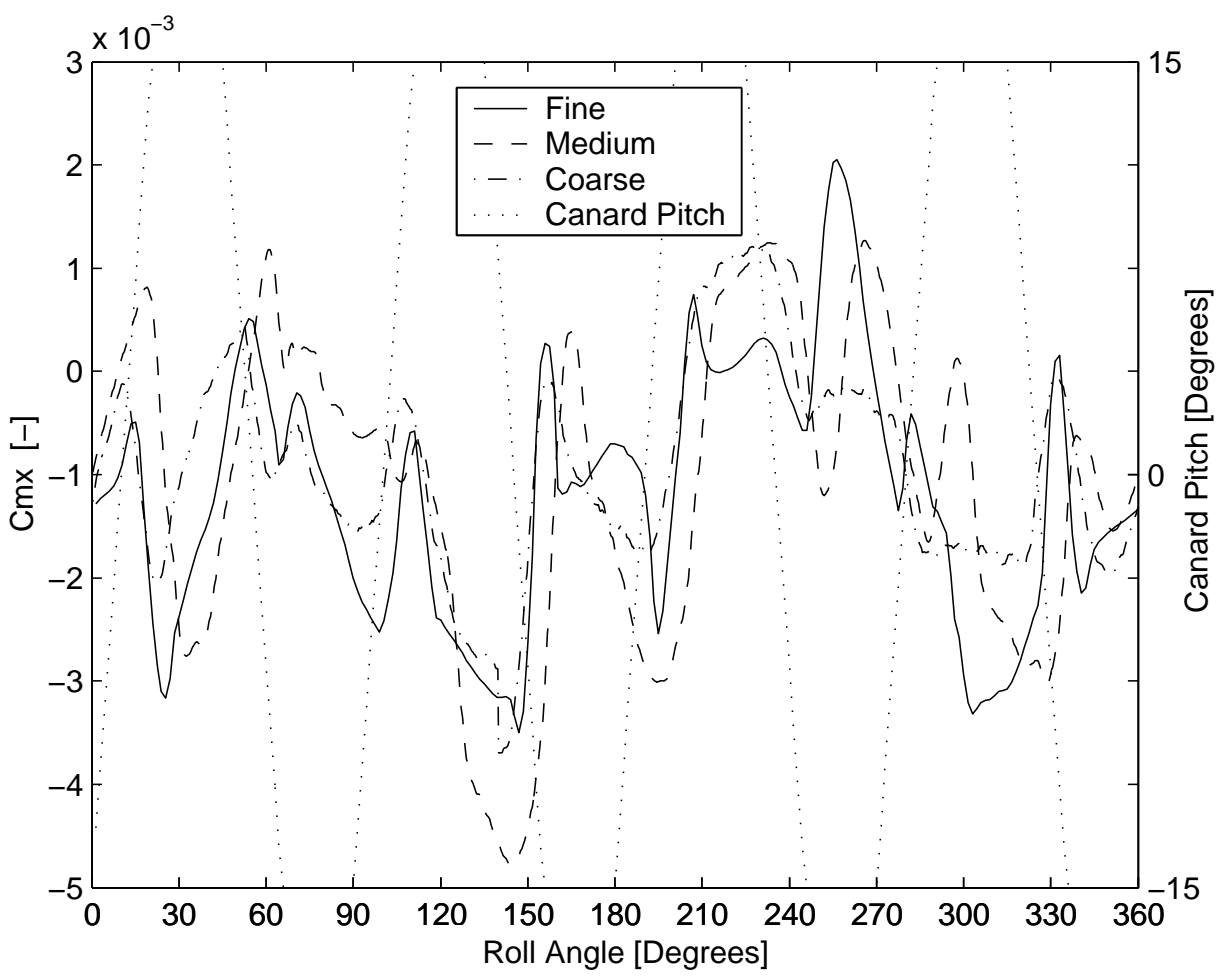

Figure 14. Grid effects in the ROLLING MOMENT coefficient.

b) V2 (medium) solution

a) V1 (fine) solution
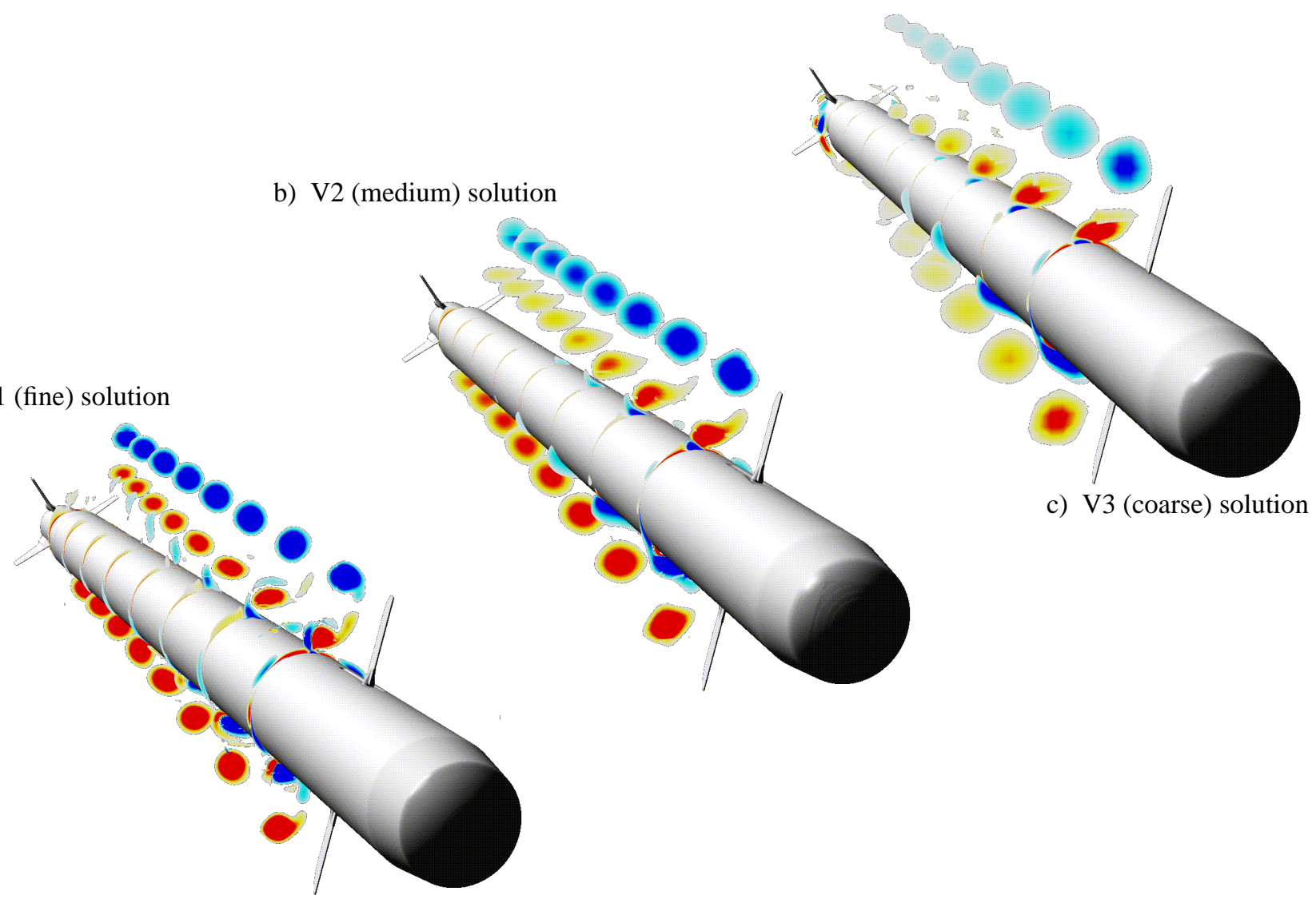

c) V3 (coarse) solution

Figure 15. Fine, medium, and coarse grid solutions for the FM-3 missile (snap-shot at a roll-angle of $268.5^{\circ}$ ). $M_{\infty}=1.6, \mathbf{R e}=50 \times 10^{7}, \alpha=3^{\circ}, \boldsymbol{c}=0 \%, \Omega_{\mathrm{r}}=8.75 \mathrm{~Hz}, \Omega_{\mathrm{d}}=35 \mathrm{~Hz}$. 
tion. The fundamental differences between static viscous and inviscid solutions are apparent and are germane to spinning cases. A spinning FM-3 missile with dithering canards case is then considered in detail for a command level of $0 \%$, free-stream angle-of-attack of $3^{\circ}$, and missile roll-rate of $8.75 \mathrm{~Hz}$.

The V1 solution considered in Section 4.1 is used as the basis of comparison for a correspondingly high resolution inviscid simulation. The high resolution inviscid, or "Euler grid," is referred to hereafter as the E1 grid system. The E1 surface grids and off-body volume grids are identical with the corresponding components of the V1 grid system. The only differences between the E1 and V1 grids is the surface normal distribution of points in the respective near-body grid components. The E1 surface normal wall spacing is 20 times that of the V1 grid. 33 million points are used to define the complete E1 grid compared to the 41 million points used in V1.

The computational savings available by assuming inviscid flow are significant. In the present high resolution cases, $20 \%$ fewer grid points are used in the E1 system than in the V1 system. Due to the larger surface normal wall-spacing, larger stable time-steps are also possible - a $\Delta \mathrm{t}$ increase of 5 times is used in the present E1 simulations, allowing for nearly 2,500 steps per missile revolution. In the present simulations, the combined effects of fewer grid points, fewer floating-point operations required per grid point, and larger time-steps result in an order of magnitude savings in computational expense.

\subsubsection{Static Geometry FM-3 Case}

Consider the qualitative differences between viscous and inviscid FM-3 missile solutions for zero rollrate and neutral canard positioning. These differences characterize some of the trends that are apparent for the spinning missile and canard dithering conditions of interest here. The vortex structure of the viscous and inviscid non-spinning FM-3 missile solutions are shown in Figure 16. The corresponding shock structure and surface pressure distributions for the two cases are shown in Figures 17 and 18, respectively.

The position and strength of the outboard canard vortices are essentially identical in both solutions. However, the inboard canard vortices differ significantly in both strength and position. Opposed by the viscous action of the boundary layer, the inboard canard vortices of the viscous solution are weaker and traverse a different path than their inviscid counterparts. The inboard canard vortices (viscous case) also pull the boundary layer off the missile surface near the tail.

At the missile nose, the shock structure is similar for the viscous and inviscid solutions. The main features are a normal shock in front of the nose, an oblique shock above and below the nose, expansion around the two sharp corners between nose and fuselage, and the shocks in front of the canards. The local influence of the canard on the fuselage pressure distribution is smeared somewhat in the viscous case, due to the boundary layer. The expansion and compression over the groove is similarly weaker in the viscous case due to the boundary layer. At the tail, the interaction between the boundary layer and the expansion-waves and shockwaves influences the pressure distribution significantly.

\subsubsection{Dynamic Geometry FM-3 Case}

Consider viscous and inviscid solutions for the spinning FM-3 missile $\left(\Omega_{\mathrm{r}}=8.75 \mathrm{~Hz}\right.$ and $\left.\Omega_{\mathrm{d}}=35 \mathrm{~Hz}\right)$. The corresponding roll-averaged force and moment coefficients are given in Table 3 . Figure 19 shows the normal force histories over one revolution. The viscous and inviscid computations agree well, except at maximum canard deflection, where there are instantaneous differences of up to $20 \%$. The influence of the deflected canards on overall forces appear more pronounced in the inviscid case, though this is an indirect effect. Figures 20,21 , and 22 decompose the normal force into contributions from the canards, fuselage, and tail section, respectively. There is almost no difference between the viscous and inviscid canard normal force histories shown in Figure 20. The over prediction of normal forces at maximum canard angles in the inviscid solution are realized from pressure distribution differences along the fuselage and tail sections. The vortex systems shed from the canards interact with the fuselage and tail fins differently in the viscous and inviscid solutions. The inviscid inboard canard vortices are too energetic and positioned incorrectly, leading to the differences seen in Figures 21 and 22. The same effects are true for the side forces shown in Figure 23.

Table 3. Roll-averaged force and moment coefs.*

\begin{tabular}{|l|c|c|}
\hline Coefficient & Viscous (V1) & Inviscid (E1) \\
\hline Cx (axial) & 1.17 & 1.10 \\
Cy (side) & $-7.56 \mathrm{e}-03$ & $-19.6 \mathrm{e}-03$ \\
Cz (normal) & 0.461 & 0.449 \\
Cmx (roll) & $-1.19 \mathrm{e}-03$ & $-1.65 \mathrm{e}-03$ \\
Cmy (pitch) & $-6.79 \mathrm{e}-03$ & $-1.06 \mathrm{e}-03$ \\
Cmz (yaw) & $2.80 \mathrm{e}-03$ & $9.17 \mathrm{e}-03$ \\
\hline
\end{tabular}

* Moments are about the missile center of gravity 

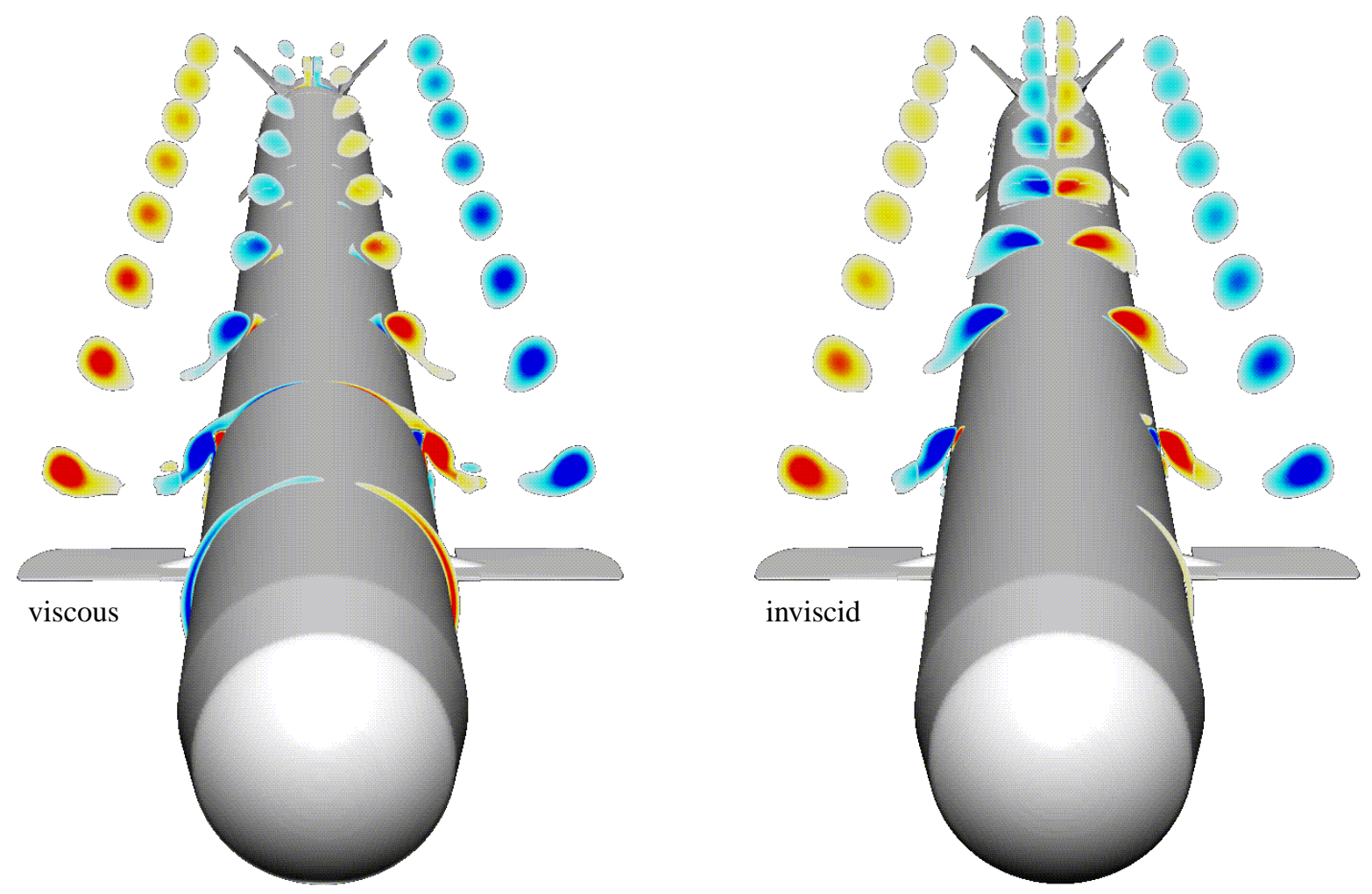

Figure 16. Vortical structure for static geometry FM-3 missile. $M_{\infty}=1.6, \mathbf{R e}=50 \times 10^{7}, \alpha=3^{\circ}$.
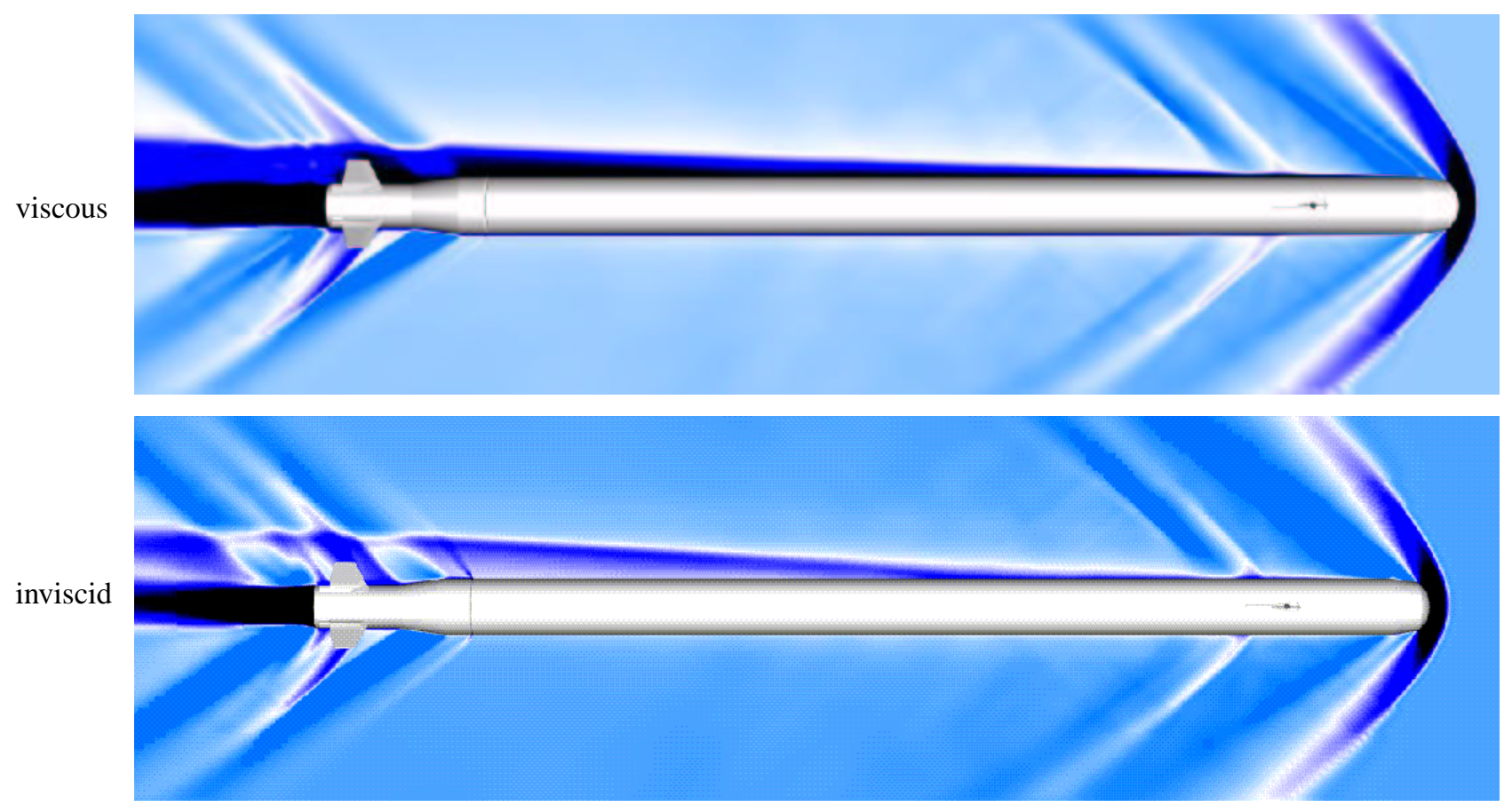

Figure 17. Shock structure for static geometry FM-3 missile. $M_{\infty}=1.6, \mathbf{R e}=50 \times 10^{7}, \alpha=3^{\circ}$. 


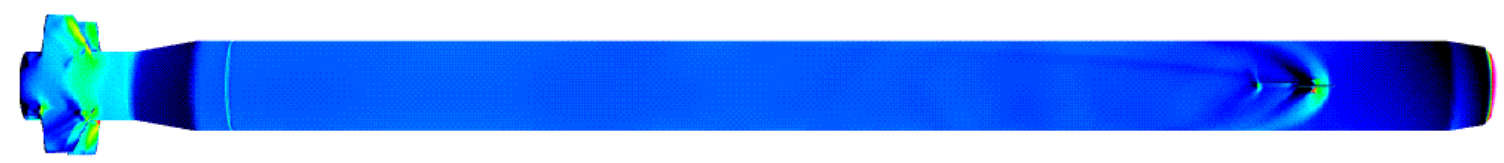

a) viscous

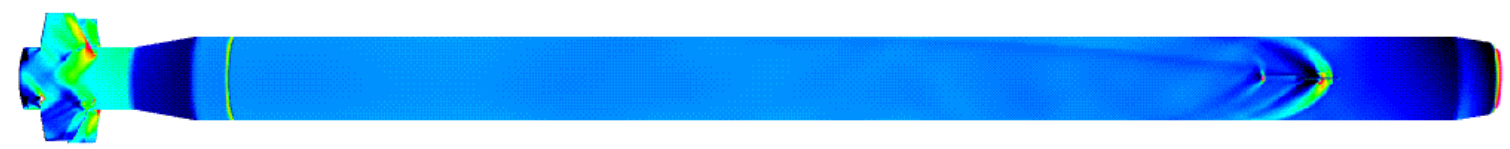

b) inviscid

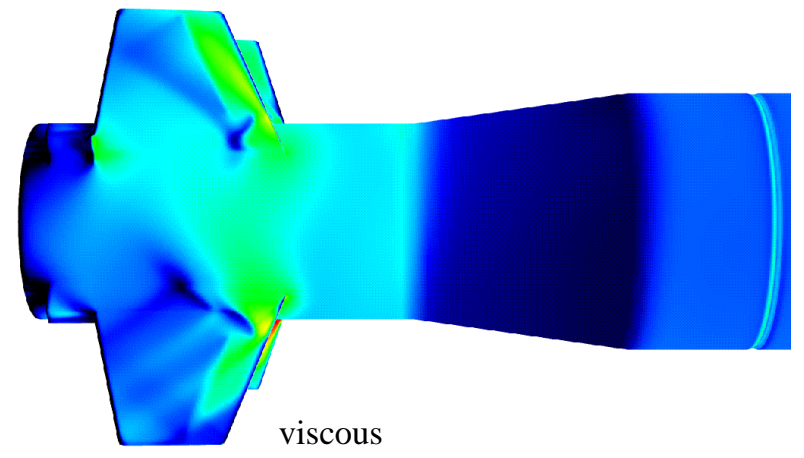

c)

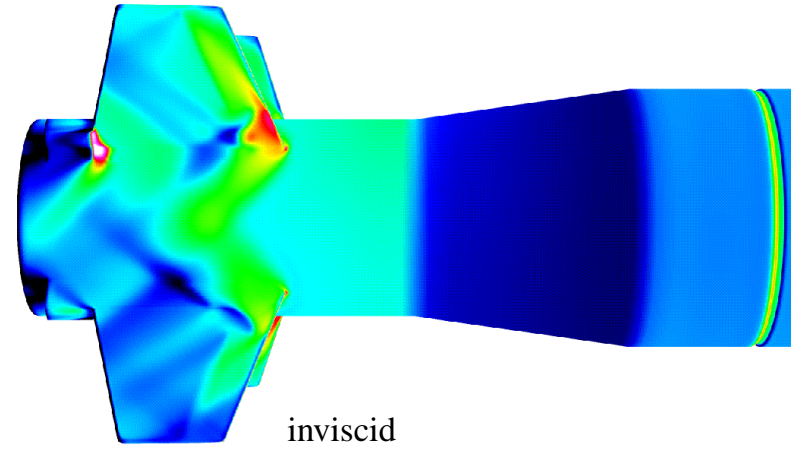

d)

Figure 18. Surface Cp distributions for static geometry FM-3 missile. $M_{\infty}=1.6, \mathbf{R e}=50 \times 10^{7}, \alpha=3^{0}$.

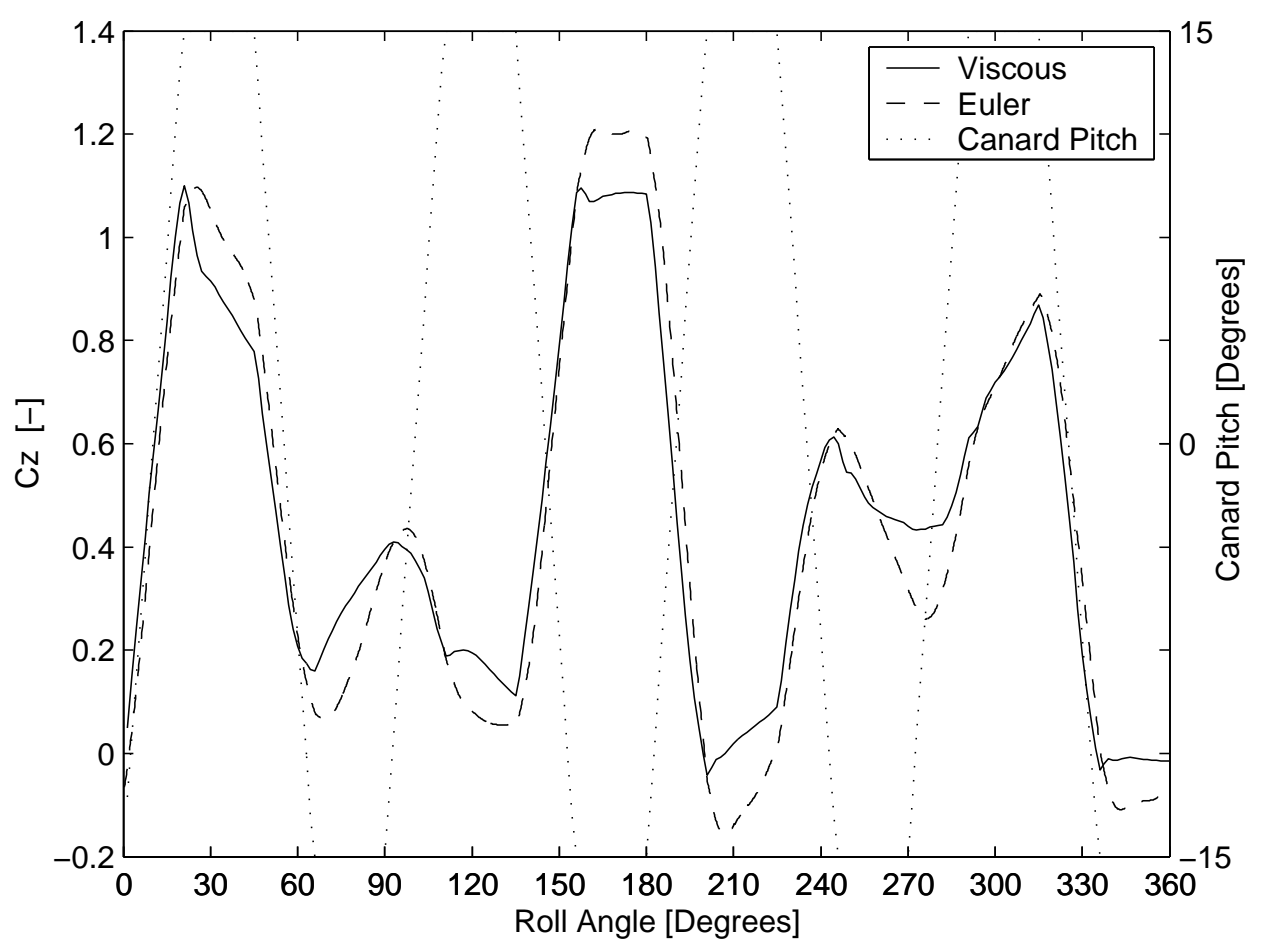

Figure 19. Viscous effects in the NORMAL FORCE coefficient. 


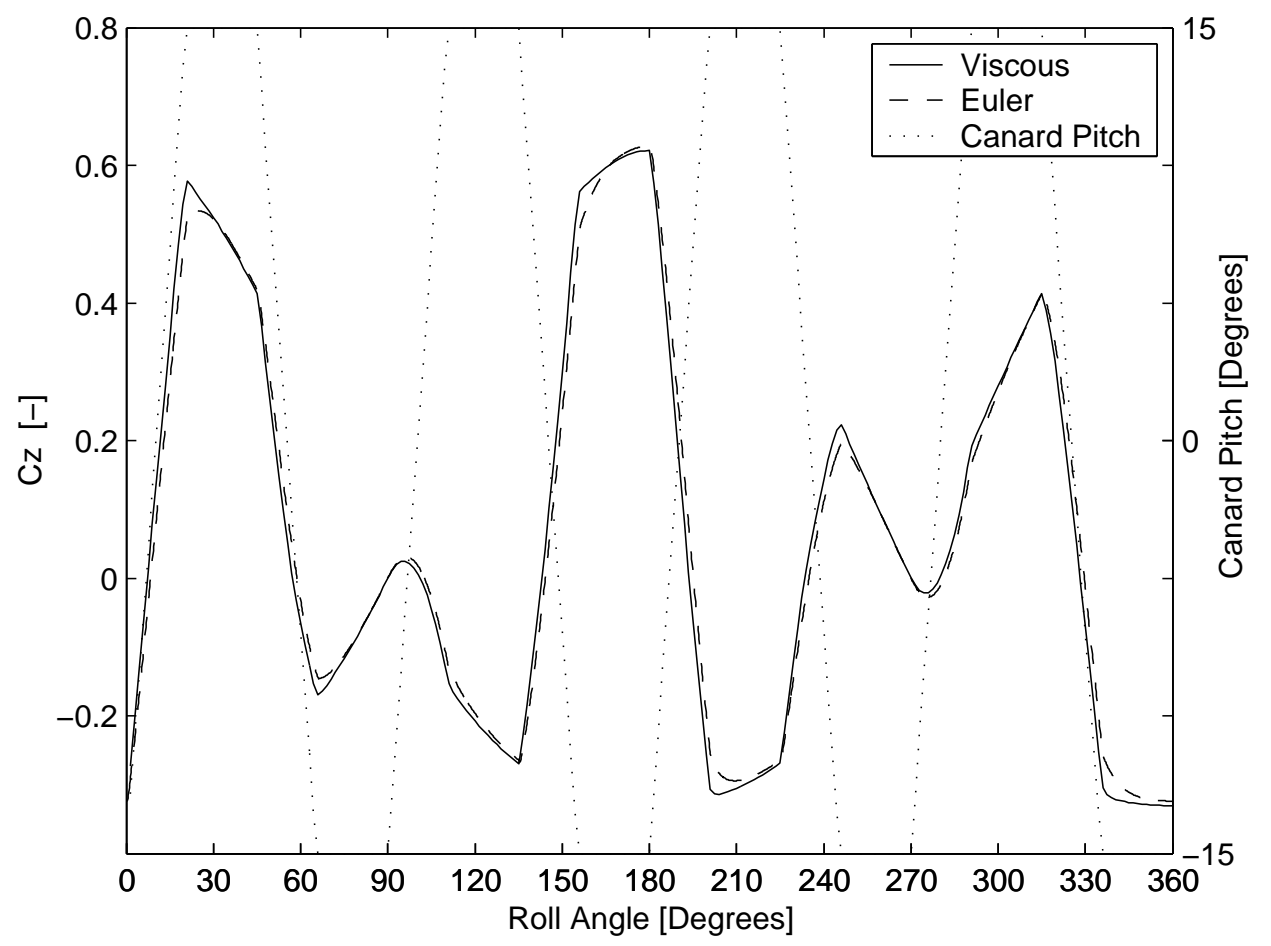

Figure 20. Viscous effects in the NORMAL FORCE coefficient (CANARD COMPONENT ONLY).

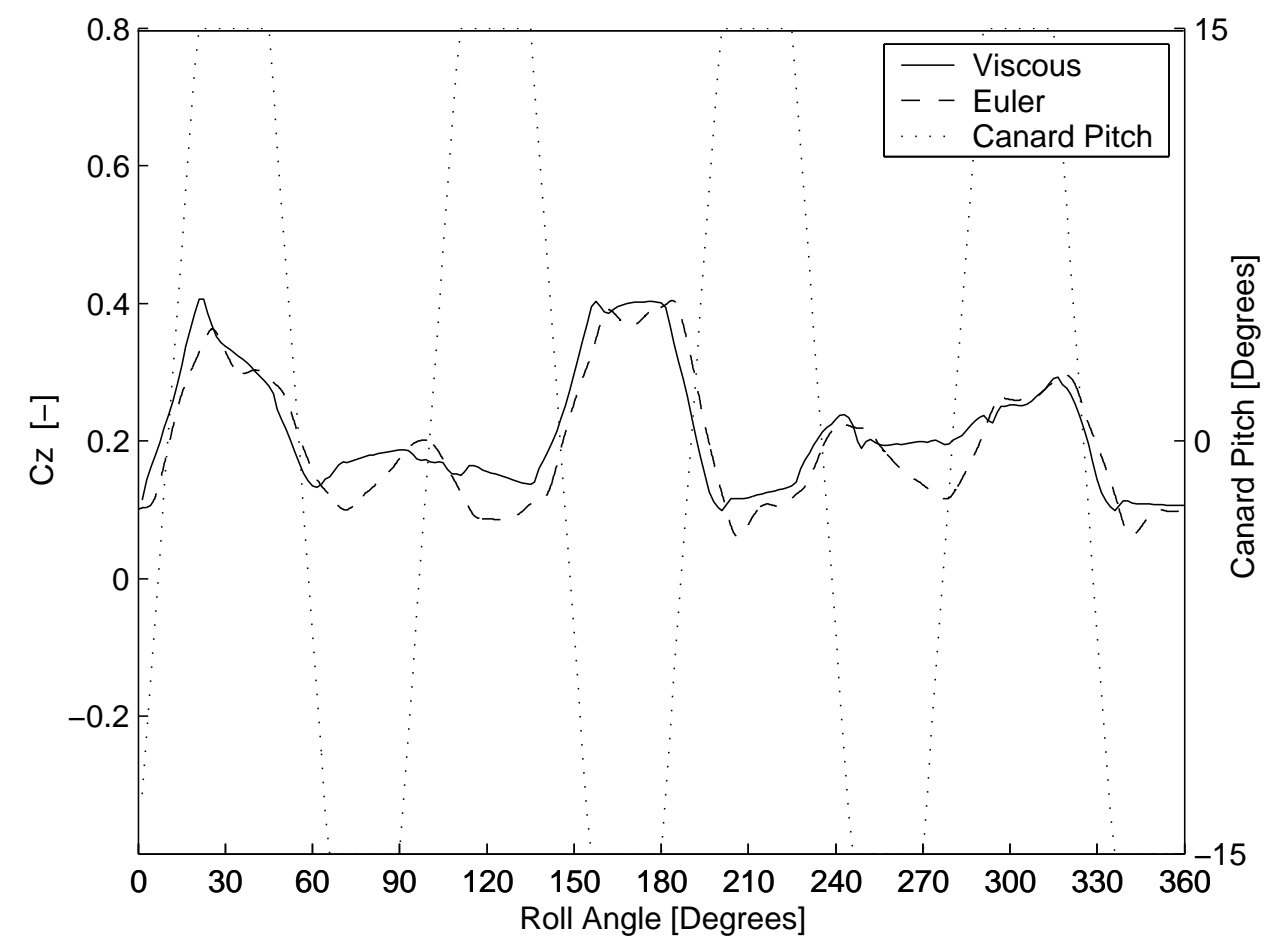

Figure 21. Viscous effects in the NORMAL FORCE coefficient (FUSELAGE COMPONENT ONLY).

American Institute of Aeronautics and Astronautics 


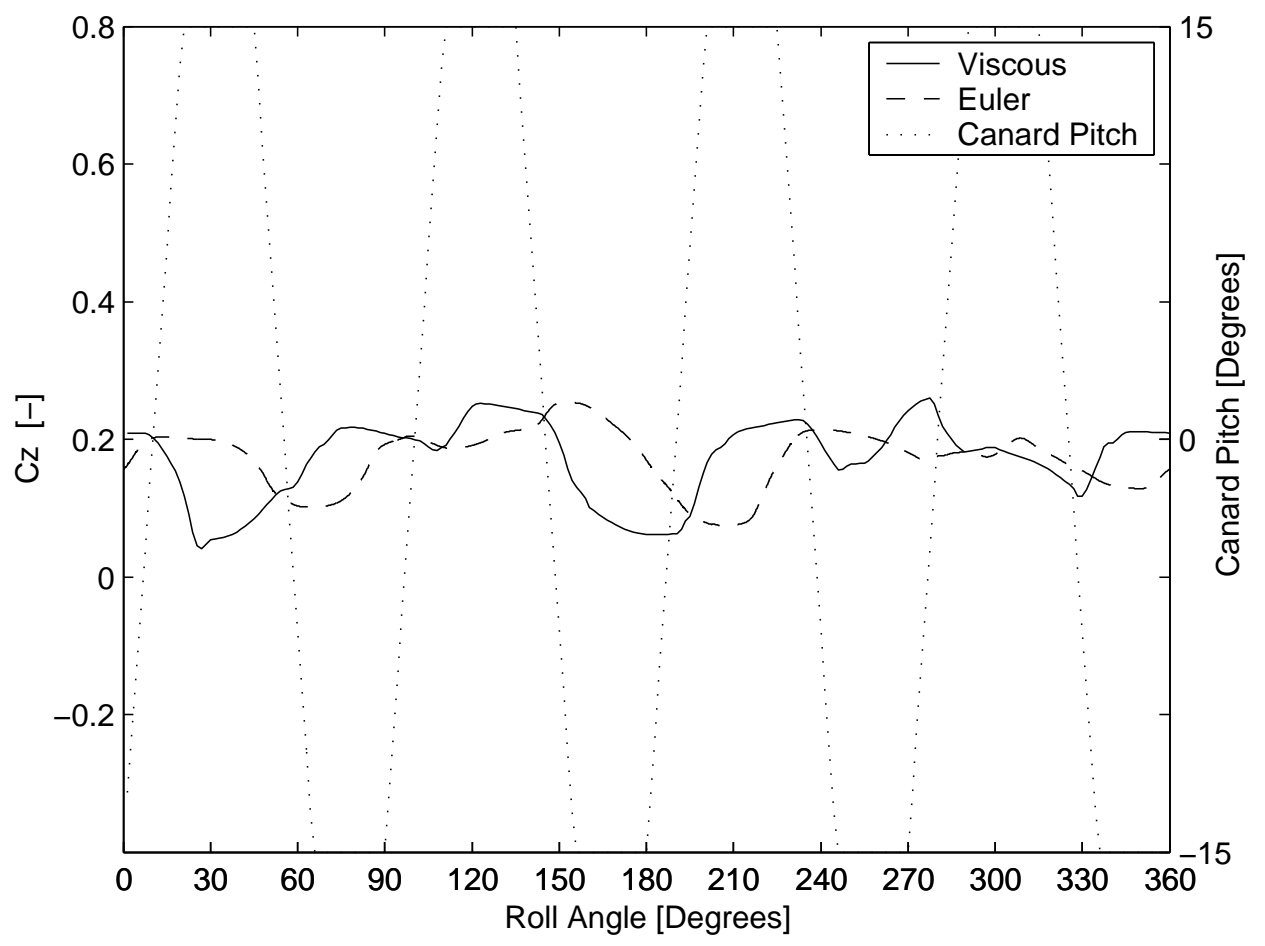

Figure 22. Viscous effects in the NORMAL FORCE coefficient (TAILFIN COMPONENTS ONLY).

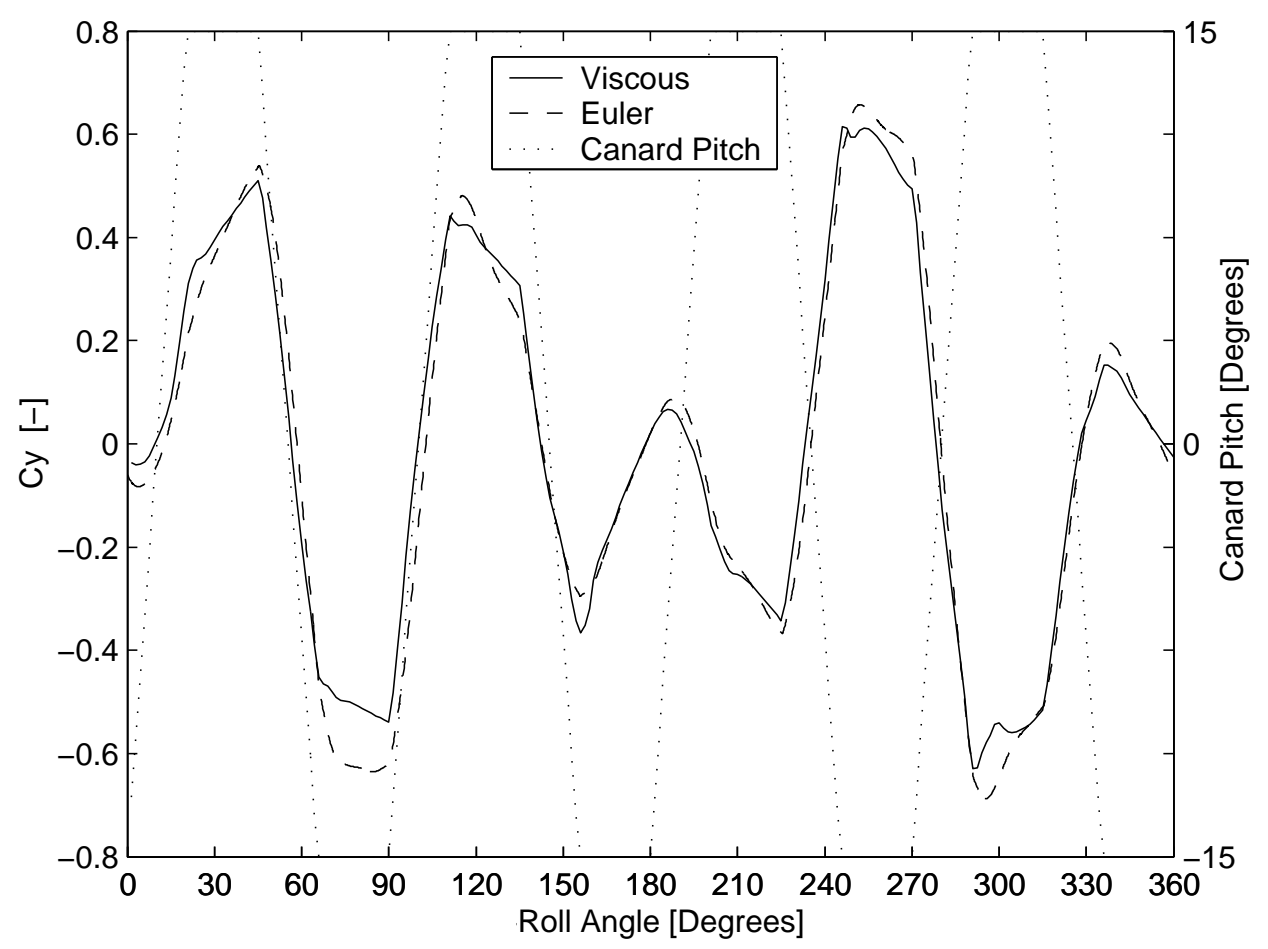

Figure 23. Viscous effects in the SIDE FORCE coefficient.

American Institute of Aeronautics and Astronautics 
The reason that the viscous and inviscid solutions agree very well everywhere except at maximum canard deflection is evident in the vortical structures shown in Figure 24. Figure 24a shows cutting planes with helicity density contours at several stations along the length of the missile for a roll-angle of $61.5^{\circ}$. At this rollangle, the canard is in transition between the maximum positive and negative canard pitch angles. Vortices trail each canard in counter-rotating pairs. Whenever the canards snap from positive to negative (or negative back to positive) pitch angle, the rotational sense of the vortices also reverse direction.

This effect is clearly evident in the instantaneous snap-shot of the vortical field shown in Figure 24a. From the canard downstream to about the missile midpoint, the vortices become weaker and finally disappear. From the midpoint back to the tail section, the vortices re-appear rotating in the opposite sense and growing slightly in strength. The history of one transition cycle is captured in this image. Vortex interaction with the boundary layer is minimal during canard transition. Accordingly, the viscous and inviscid solutions are in very good agreement for all roll-angles where the canards are in transition.

Figure $24 \mathrm{~b}$ shows cutting planes with helicity density contours at several stations along the length of the missile for a roll-angle of $84^{\circ}$. The instantaneous solution indicated in the figure corresponds to the completion of nearly 20 degrees of missile role, or 2.5 missile body-lengths of travel, with the canards positioned at maximum negative deflection. The fully developed canard vortices prevail well past the missile. The vortices interact with each other and with the boundary layer. The inboard canard vortices are weakened by the boundary layer, reducing the force peaks, as seen in the normal and side force histories of Figures 19 and 23. The inboard canard vortex/boundary layer interactions are entirely unaccounted for in an inviscid simulation.

The roll-averaged axial force for the viscous solution is about $8 \%$ higher than the corresponding inviscid solution (see Table 3). Figure 25 shows the corresponding axial force histories over one revolution. Approximately $60 \%$ of the difference noted between the viscous and inviscid axial force is due to viscous wall stresses. The remaining $40 \%$ of the difference is due to spatial variations in the surface pressure distributions. The pressure contribution to axial force in the viscous solution is compared separately in Figure 26 with the inviscid axial force. Boundary layer/shock interactions in the viscous solution lead to differences in overall shock structure and surface pressure distributions, accounting for these differences.

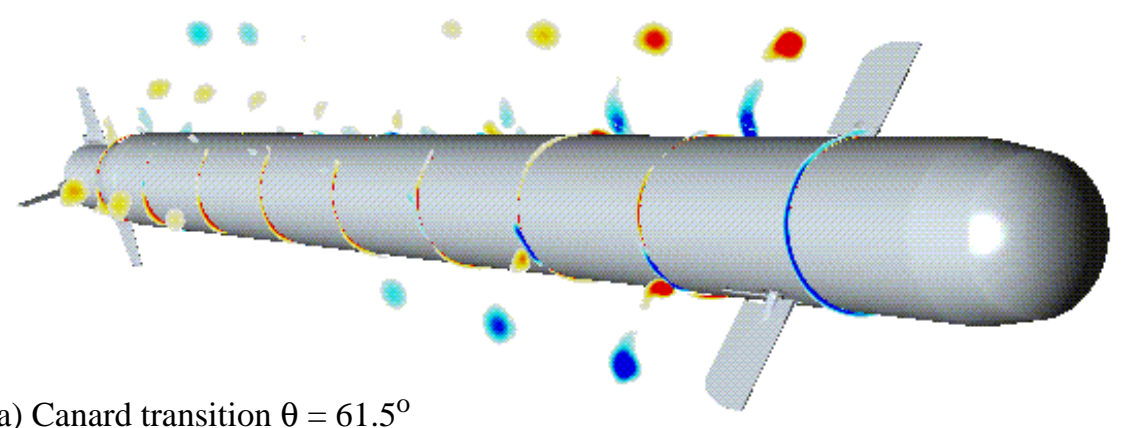

a) Canard transition $\theta=61.5^{\circ}$

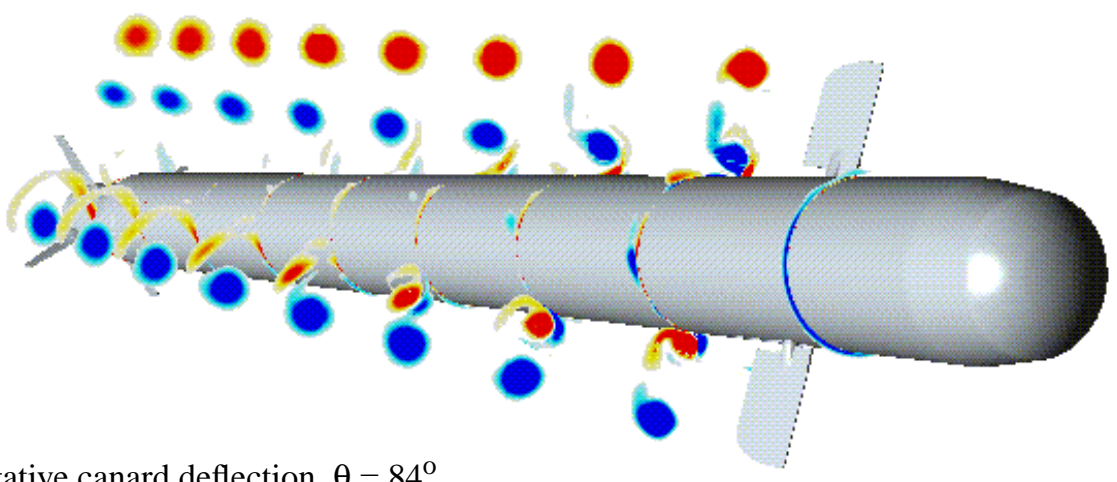

b) Maximum negative canard deflection, $\theta=84^{\circ}$

Figure 24. Vortical structure for vsicous FM-3 spinning missile case. $M_{\infty}=1.6, \mathbf{R e}=50 \times 10^{7}, \alpha=3^{\circ}, c=0 \%$, $\Omega_{\mathrm{r}}=8.75 \mathrm{~Hz}, \Omega_{\mathrm{d}}=35 \mathrm{~Hz}$. 


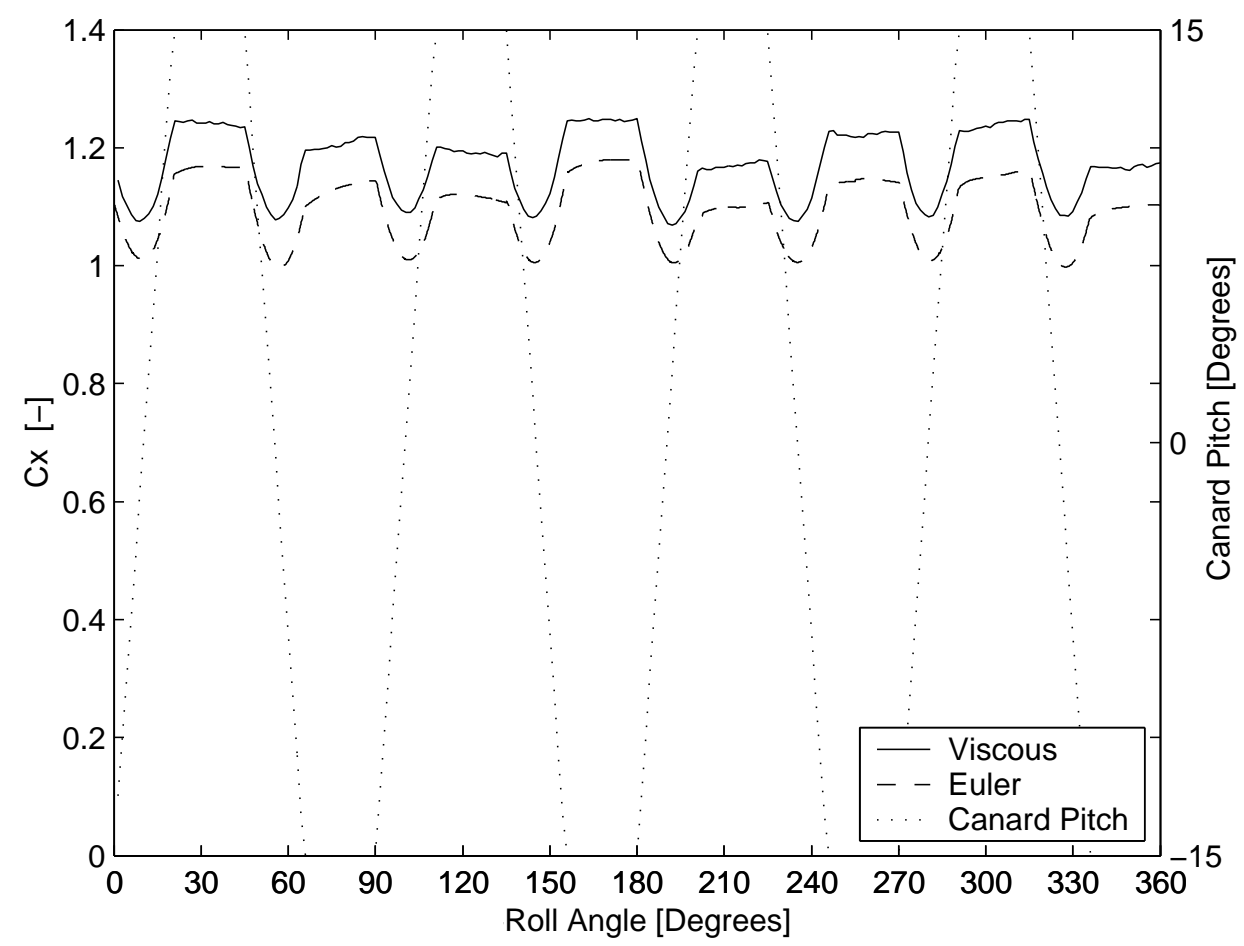

Figure 25. Viscous effects in the AXIAL FORCE coefficient.

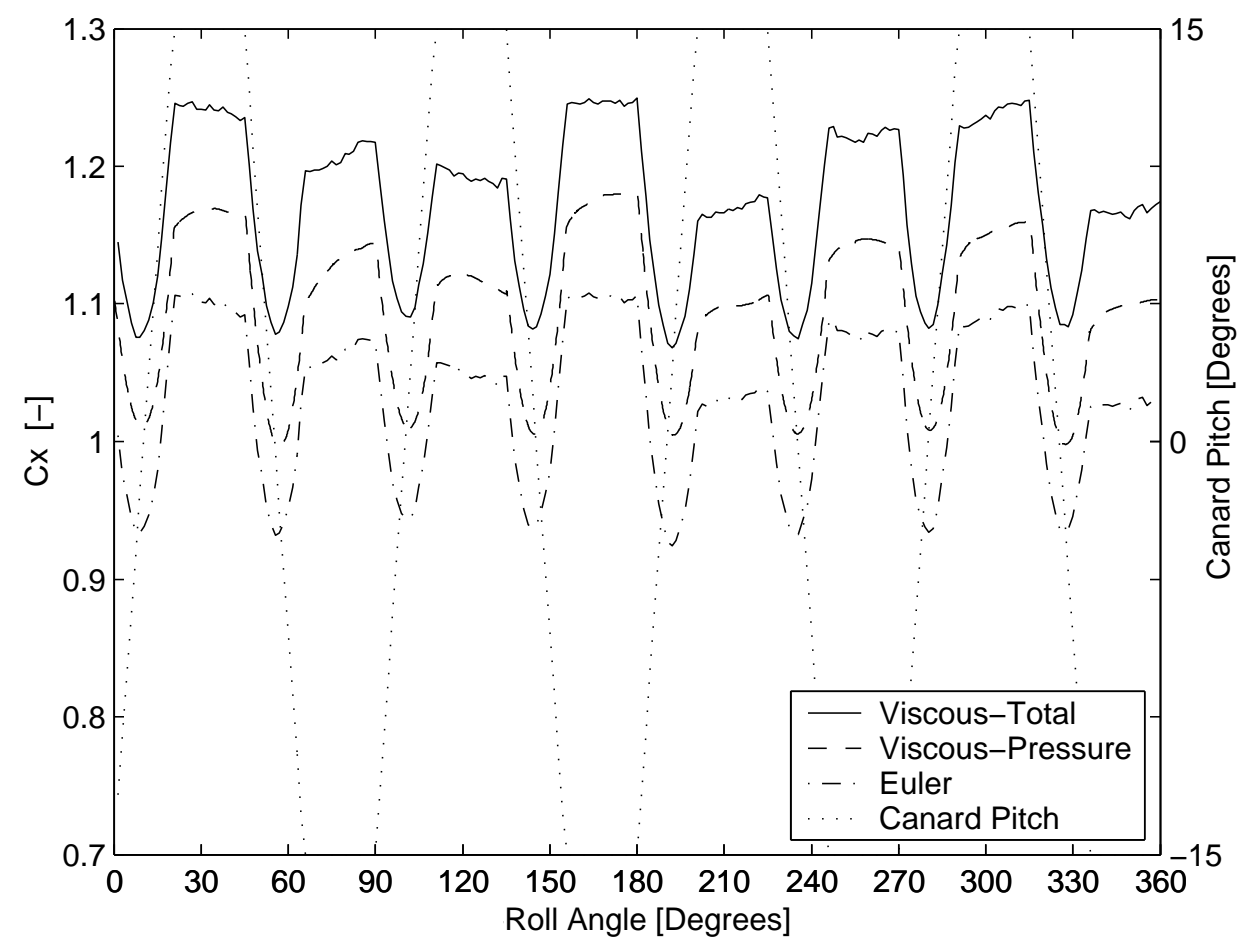

Figure 26. Viscous effects in the AXIAL FORCE coefficient. 
Figure 27 shows the pitching moment about the missile center of gravity. The viscous solution has higher peaks. The stronger vortices in the inviscid solution interact with the fuselage and tail fins, adding a moment that counteracts the moment from the canards. A similar effect can be seen for the yawing moment in Figure 28.

Based on the high resolution viscous (V1) and inviscid (E1) simulations, a few statements regarding the relative significance of viscous effects on FM-3 missile performance are justified.

$i$. Instantaneous side- and normal forces differ up to $20 \%$. This is due to differences in interaction between the vortex system shed from the canards and the fuselage boundary layer and tail fins.

ii. The axial forces differ by about $8 \%$. The viscous wall stresses accounts for $60 \%$ of this difference.

iii. Euler computations provide valuable information, but viscous effects should ideally be included in detailed studies.

\subsection{Roll-Rate Effects}

An important objective in considering the utility of computational methods (and physical experiments) for rolling airframe missile systems is the accurate prediction of roll-averaged forces and moments. A large parameter space can easily exhaust computational (or experimental) resources, making it important to eliminate parameters of secondary importance. The spinning missile cases defined in Table 1 have a roll-rate of 8.75 $\mathrm{Hz}$, which allows for only $8^{\mathrm{O}}$ of missile roll per bodylength of travel. It seems plausible that for a given pitching sequence and moderate angular velocity, the roll-averaged forces may not be sensitive to roll-rate. The relative significance of roll-rate effects in this regime are determined here by evaluating differences between solutions having roll-rates of zero and $8.75 \mathrm{~Hz}$. The baseline flight conditions defined in Table 1 and the V2 grid system are used in all cases. The canard command level is $0 \%$ and the free-stream angle-of-attack is $3^{\mathrm{o}}$.

The $8.75 \mathrm{~Hz}$ spinning missile case is discussed at length in Section 4.1 and results using the V2 grid system are given in Table 2 and Figures 7 through 15. The "zero" roll-rate cases considered in the present section for comparative purposes are generated in a discrete quasi-static fashion for the same roll-angle and canard dither cycle. The quasi-static results for the zero roll- rate condition can be obtained in one of several ways. Two obvious ways are as follows.

i. $\quad$ Static geometry. Start with the desired missile roll and canard pitch angles fixed in freestream conditions. Drive the corresponding solution to convergence in steady-state mode. Switch to time-accurate mode (static geometry) and continue the solution to determine the temporal state of the flow.

ii. Frozen geometry. Freeze the geometry of the $8.75 \mathrm{~Hz}$ spinning case at the desired missile roll and canard pitch angles. Initialize the solution with the corresponding instantaneous spinning result obtained previously (see Section 4.1). Hold the roll and canard pitch angles constant and run the simulation time-accurately until the false-transient dies and to determine the temporal state of the flow.

Figure 29 shows the time-history of the normal force that results from the "static geometry" and "frozen geometry" approaches corresponding to a roll-angle of $150^{\circ}$ and $-6.95^{\circ}$ canard pitch angle. The normal force coefficient is plotted versus "relative" time-step, because both steady-state and time-accurate integration are used during the convergence histories. For the static geometry case, the solution is run to convergence in steady-state mode. At relative time-step zero, the timeintegration scheme is switched to time-accurate mode and run further as shown. In all, more than 15,000 timesteps are needed to obtain a converged solution with this approach. The number of time-steps (or iterations) can be reduced with alternative integration schemes (e.g., dual time-stepping, multigrid, etc.), but the effort is still a significant fraction of the cost of computing a full revolution for a dynamic case.

In the frozen geometry result shown in Figure 29, the instantaneous solution from the spinning missile case is used for initial conditions. At relative time-step zero, the missile orientation is frozen to the identical state used with the static geometry approach and the solution is commenced time-accurately. Cz quickly settles (in less than 500 steps) to a value that is identical to the $\mathrm{Cz}$ obtained from using the static geometry approach. Time histories for the other forces and moments show similar behavior. A comparison of the two methods can be summarized as follows:

$i$. The two methods provide the same results for forces and moments.

ii. A restart from the dynamic flow-field provides a steady-state solution at least 20 times faster than starting from scratch for this particular roll-rate. 


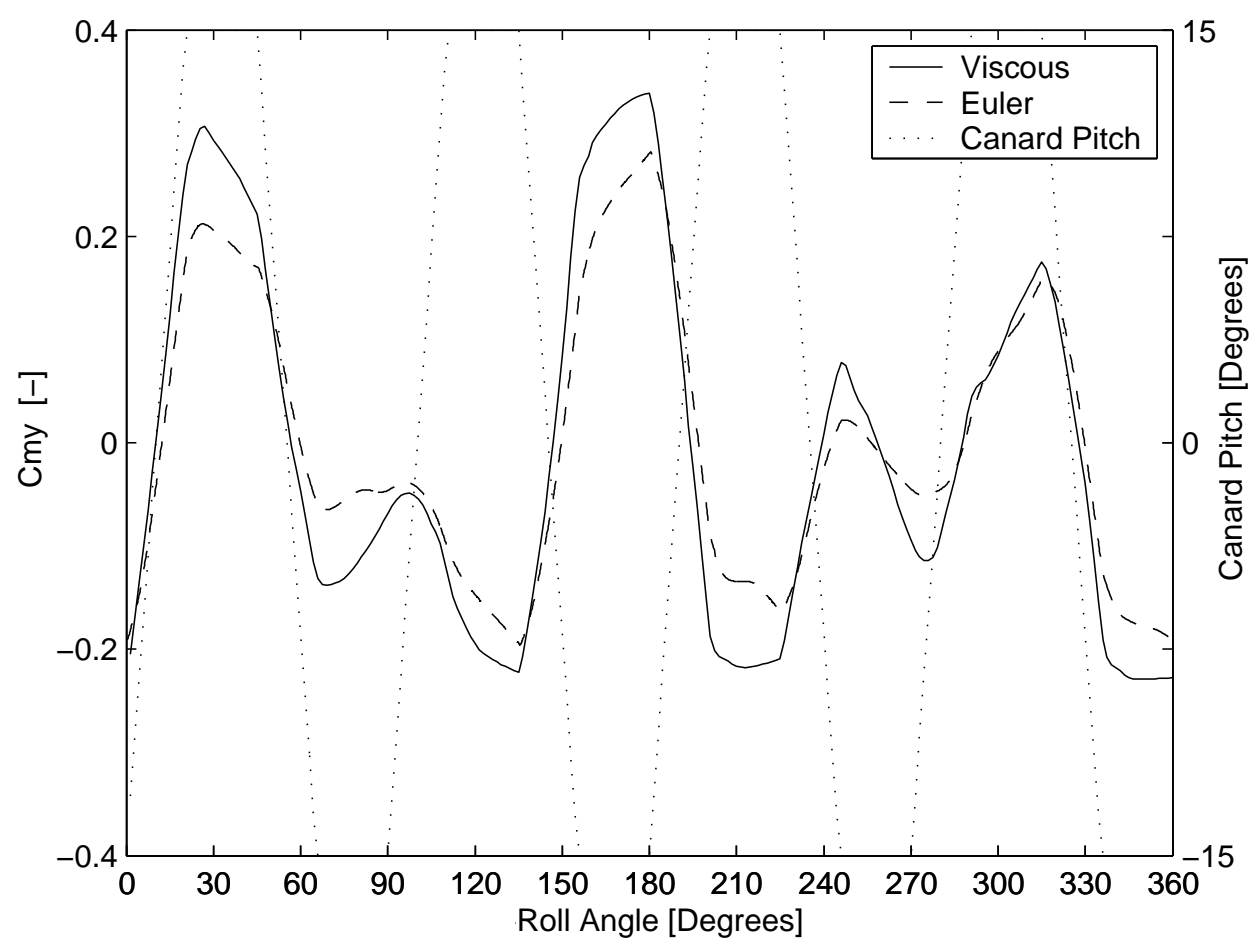

Figure 27. Viscous effects in the PITCHING MOMENT coefficient.

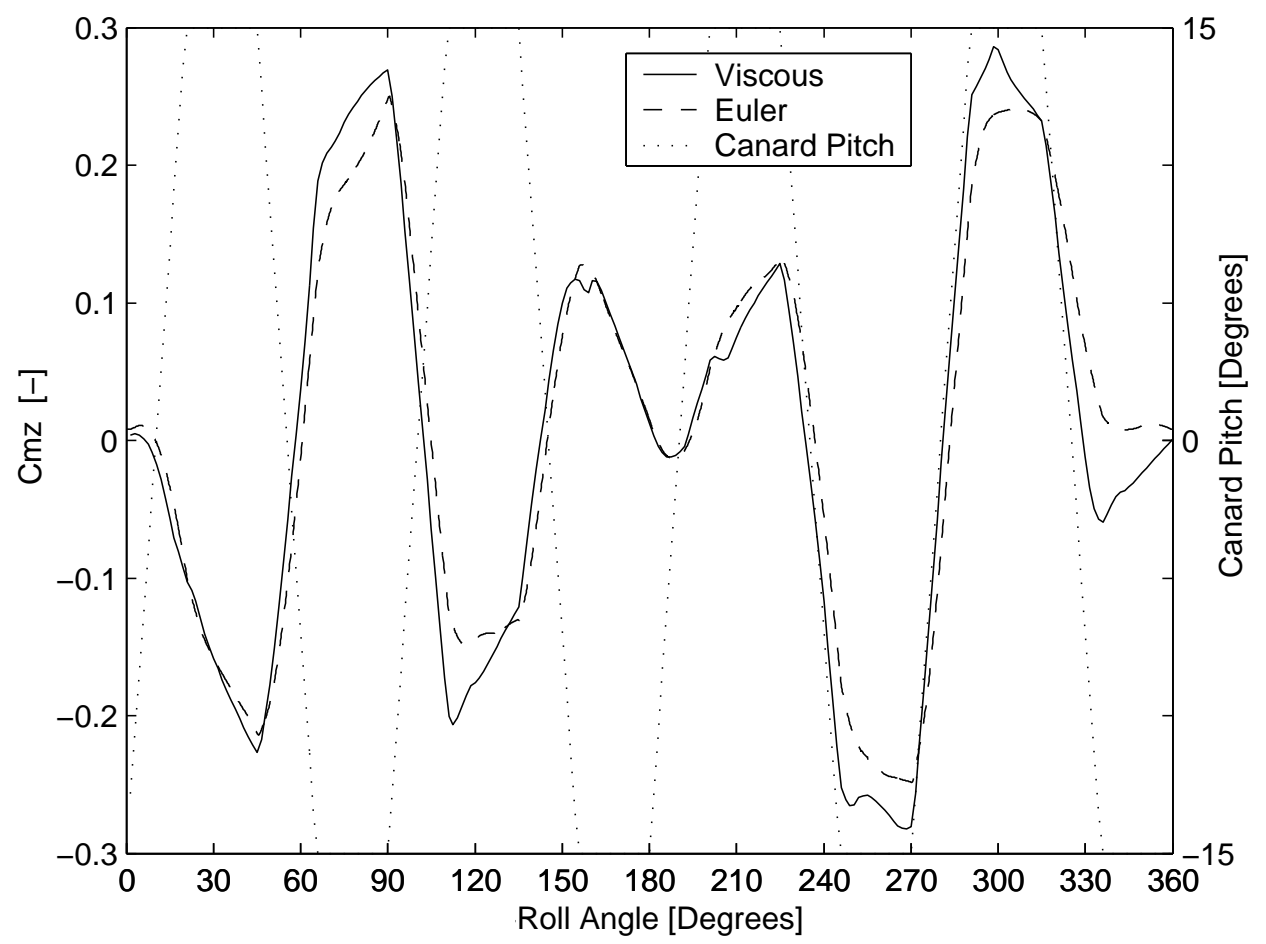

Figure 28. Viscous effects in the YAWING MOMENT coefficient.

American Institute of Aeronautics and Astronautics 


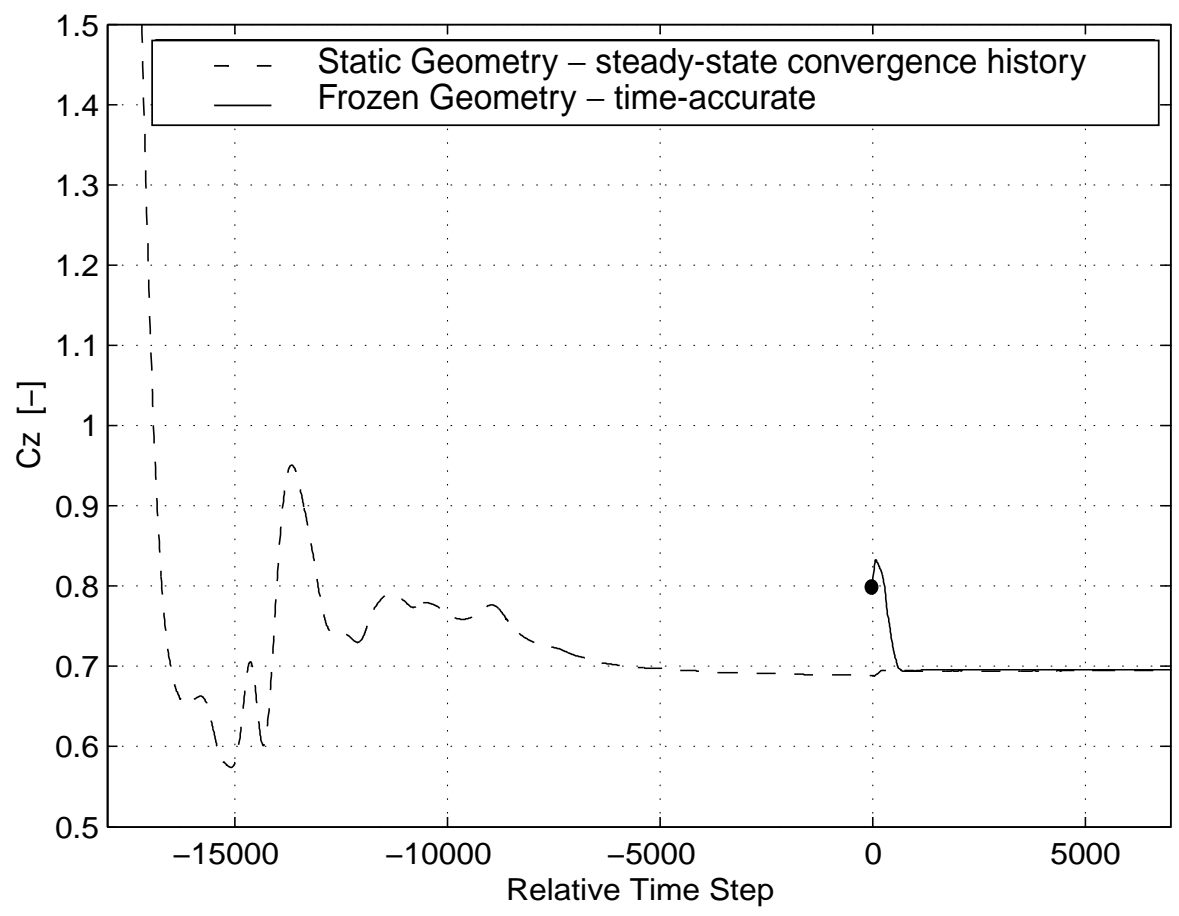

Figure 29. NORMAL FORCE coefficient. Comparison of "static geometry" and "frozen geometry" approaches to generating quasi-static solutions for rolling airframe missiles. Static geometry converged in steady-state mode until step 0 , then run time-accurately. Frozen geometry case run time-accurately from spinning missile initial condition.

iii. Even if the problem turns out to be quasisteady, it is more efficient to run through a dynamic computation rather than computing a series of static solutions.

The difference between the frozen geometry initial solution and the converged static geometry solution directly shows the point-wise relative effect of roll-rate. Figure 30 shows a comparison of 6 static solutions (obtained via the frozen geometry method) to the timeaccurate solution for the $8.75 \mathrm{~Hz}$ spinning case. The corresponding static and dynamic results are in good agreement. For roll angles $30^{\circ}, 90^{\circ}, 210^{\circ}$ and $270^{\circ}$, the dynamic results lag the static results by a few degrees of roll angle. For the roll angles $150^{\circ}$ and $330^{\circ}$, the canard is in the middle of a canard "snap" between the two extreme deflections. This causes a dynamic inflow effect, with both a delay and larger changes of forces on the canards than what is sustained in a static configuration of the missile. At $150^{\circ}$ roll angle, the force overshoot outweighs the lag effect, and shifts the dynamic solution ahead of the static solution. At a roll angle $330^{\circ}$, the effects seem to cancel.

The other forces and moments (except rolling moment) show similar behavior. The rolling moments differ significantly. This is not surprising, since the rolling moment is generated by the tail fins for which the vorticity dynamics become significant. Since the V1, V2, and V3 grid resolution study does not demonstrate convergence for the rolling moment, an analysis of rollrate effects on rolling moment is not attempted here.

In a study of the significance of roll-rate effects on the FM-3 missile aerodynamics, six data points can only give an indication of the complete phenomenon. Still, it seems plausible that moderate changes of roll-rate in the range of $0-10 \mathrm{~Hz}$ should not affect roll-averaged forces significantly.

\subsection{Computational Expense}

The FM-3 missile cases converge to a periodically repeating solution in approximately $460^{\circ}$ of roll. The computational expense of each V2 time-accurate solution is approximately 438 hours of CPU time on a 300 $\mathrm{MHz}$ processor (viz., SGI Origin 2000) per revolution, or 560 CPU hours per case. Most of the V2 results presented in this paper are the result of runs using 16 processors, with a processing rate of 35 hours per case and approximately $95 \%$ parallel efficiency.

The OVERFLOW-D performance rate realized for the V2 solutions justifies the contemplation of aerodynamic database population with Navier-Stokes solutions. Maximum parallel efficiency per case is realized 


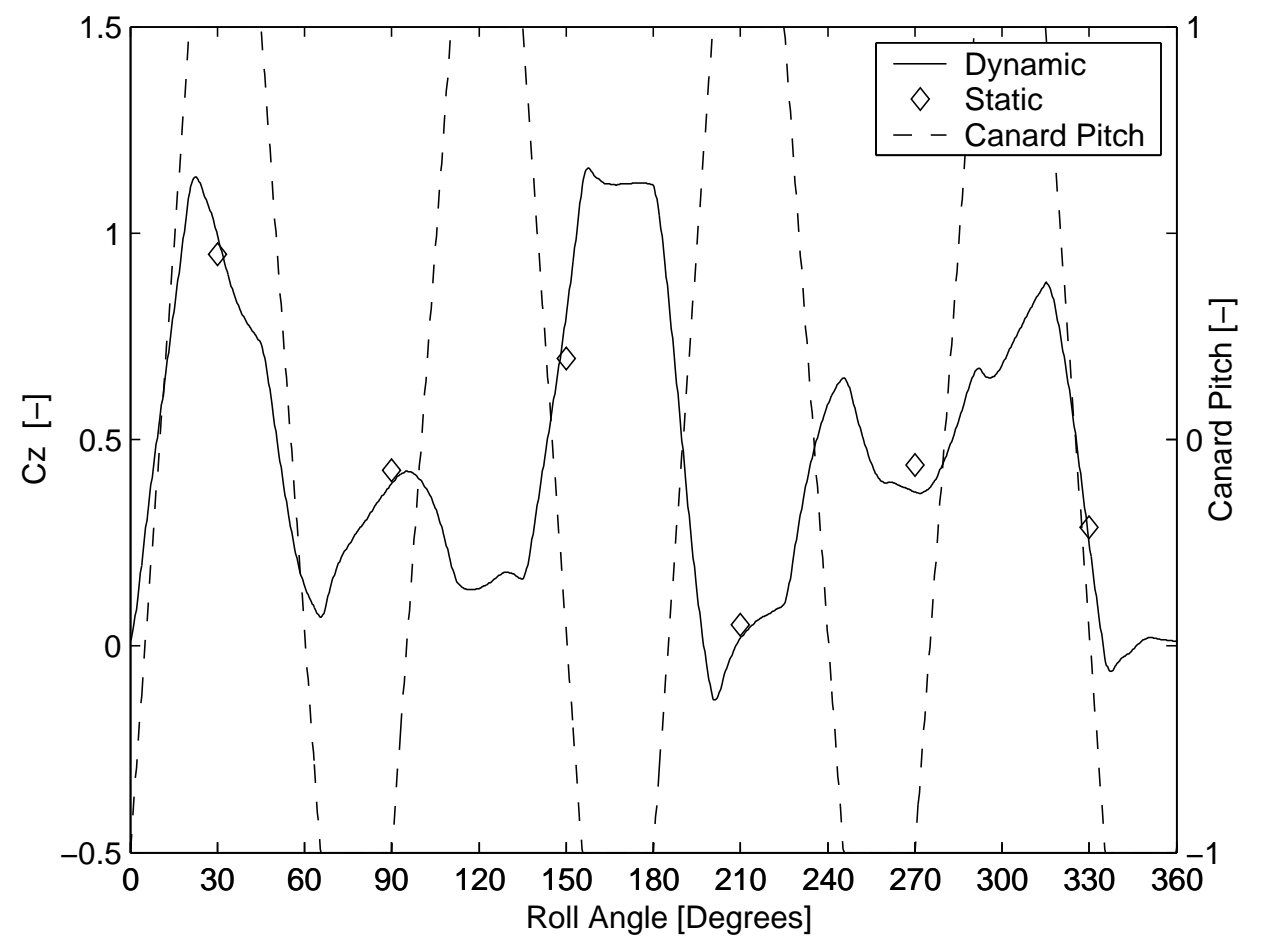

Figure 30. Roll-rate effects in NORMAL FORCE coefficient.

when the minimum number of processors that will accommodate the case in core memory is used. Four processors are sufficient for the V2 solutions (assuming 1 gigabyte of memory per processor). Simultaneous computation of multiple cases leads to perfect parallelism in the generation of solution sets. For example, a 256 node SGI Origin 2000 can execute 64 FM-3 cases simultaneously yielding a throughput of approximately 330 cases per month. A database populated by conventional means (i.e., physical experiments) does not require many more points than this. For a rolling airframe, such as the FM-3, approximately 600 spinning missile data points is sufficient for a parameter space that includes Mach number, angle-of-attack, command level, and roll-rate.

\section{AERODYNAMIC PERFORMANCE}

\section{$\underline{5.1 \text { Simulation Results }}$}

Given an understanding of the grid resolution requirements and the relative significance of viscous and roll-rate effects, a matrix of cases is defined to evaluate the aerodynamic performance of the FM-3 spinning missile subject to variations in free-stream angle-ofattack $(\alpha)$ and canard command level $(\boldsymbol{c})$. The baseline conditions for the case matrix are defined by the fixed parameters of Table $1\left(M_{\infty}, \mathbf{R e}\right.$, and $\left.\Omega_{\mathrm{d}}\right)$ and with the roll-rate $\left(\Omega_{\mathrm{r}}\right)$ set to $8.75 \mathrm{~Hz}$. The matrix consists of 21 unique cases defined by varying $\alpha$ and $c$ over the ranges indicated in Table 1 (viz., $\alpha=0^{\circ}, 2^{\circ}, 3^{\circ}, 4^{\circ}, 8^{\circ}, 12^{\circ}, 15^{\circ}$ and $\boldsymbol{c}=0 \%, 100 \%, 200 \%$ ). The pitching sequences that result from the three command levels are shown in Figure 31 .

The results given in Section 4 demonstrate that although inviscid computations can provide valuable information about the FM-3 spinning missile, there are significant viscous effects (e.g., fuselage boundary layer damping of the inboard canard vortex). The results also indicate that the medium resolution viscous grid (V2) yields comparable results to the 41 million point baseline viscous grid (V1). Accordingly, the case matrix considered here is populated entirely with viscous solutions using the V2 grid system. The results of Section 4 also suggest that roll-rate effects are not important for the parameter space of interest here. However, the cost of generating 21 time-accurate spinning missile solutions to populate the case matrix is far less expensive than generating a comparable data base composed of quasi-static solutions. Time-accurate simulation data is therefore used.

The normal forces for the 7 cases with command level $0 \%$ are shown in Figure 32. The corresponding results for the $100 \%$ and $200 \%$ command level are shown in Figures 33 and 34, respectively. Results for 


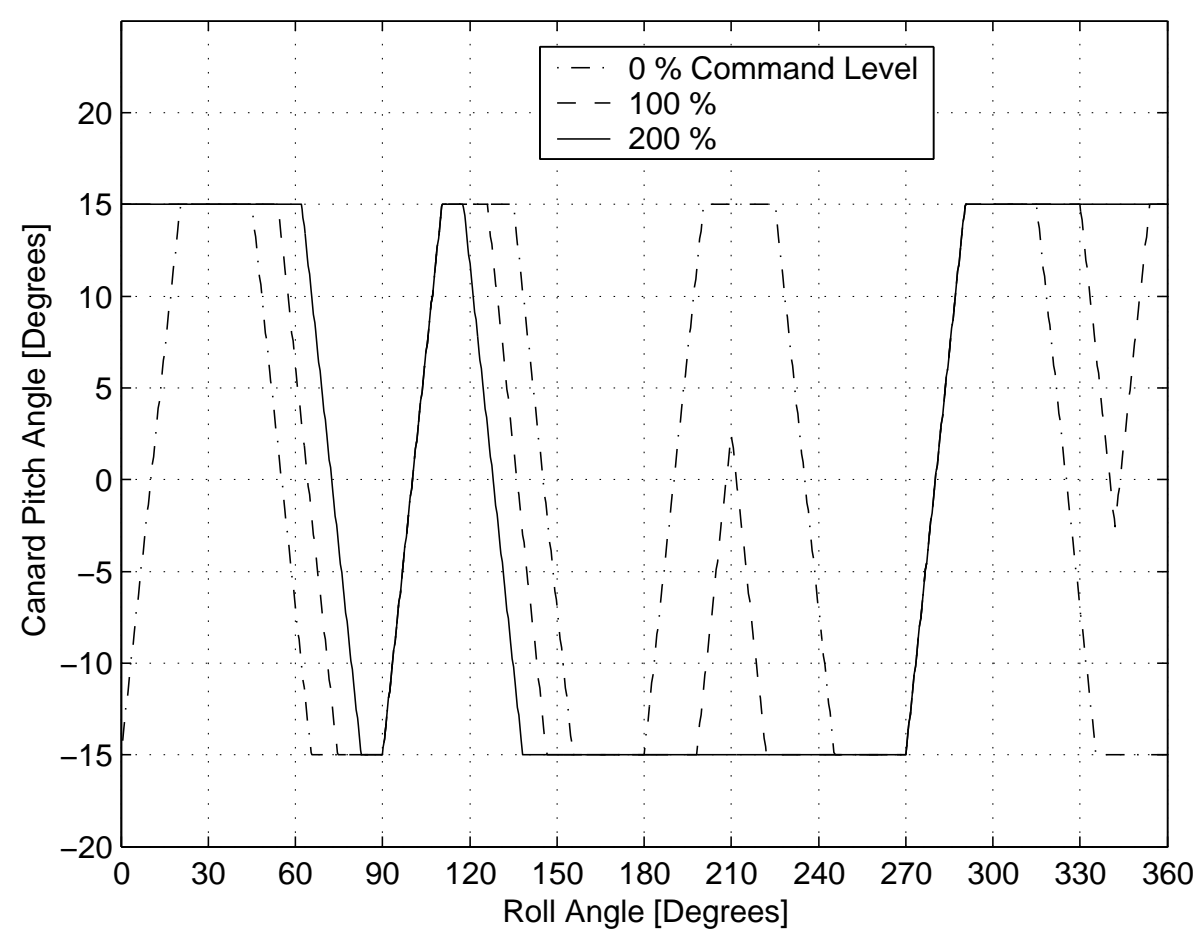

Figure 31. Canard pitching sequences for $0 \%, 100 \%$, and 200\% command level.

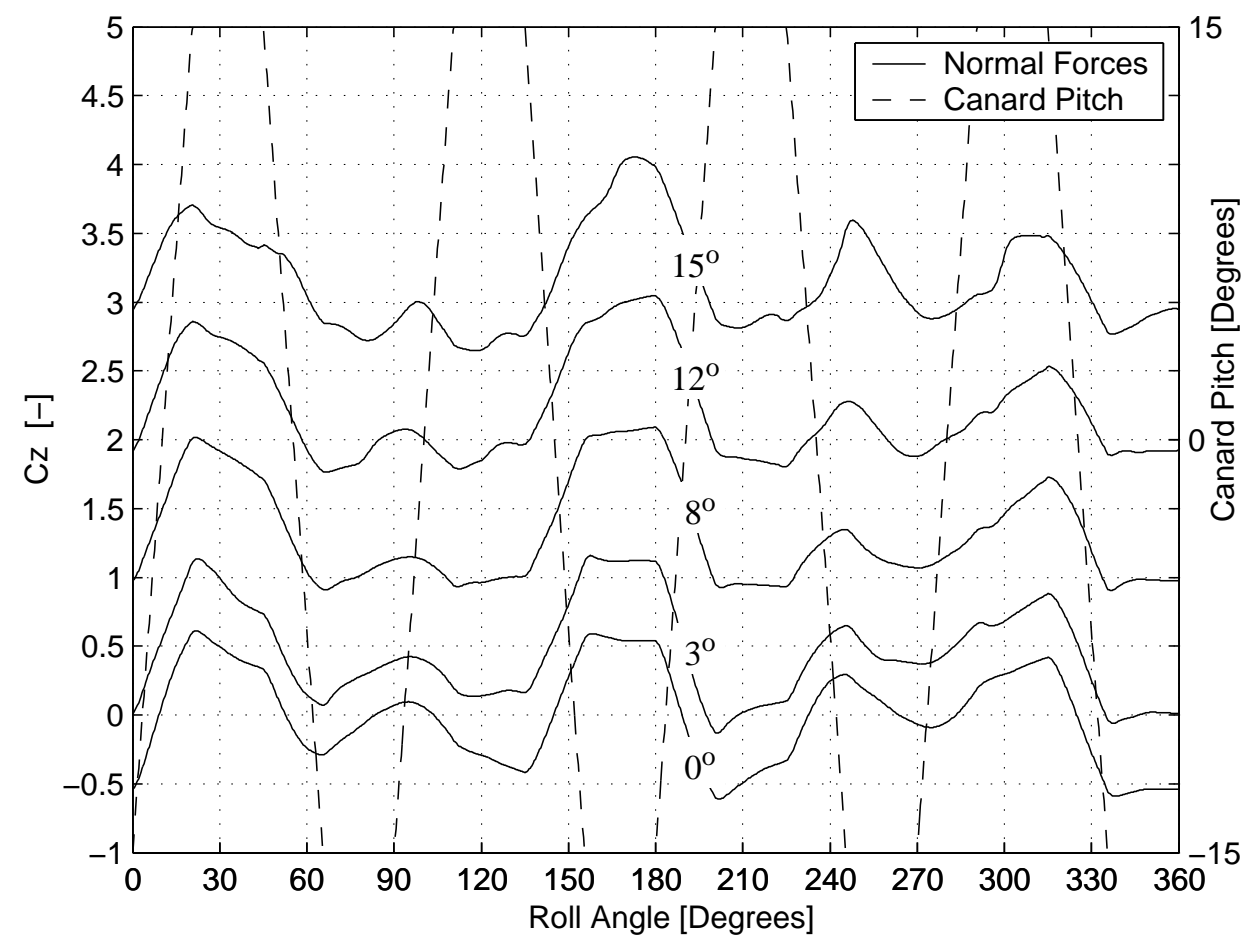

Figure 32. NORMAL FORCE coefficient for different $\alpha$ at $0 \%$ command level. 


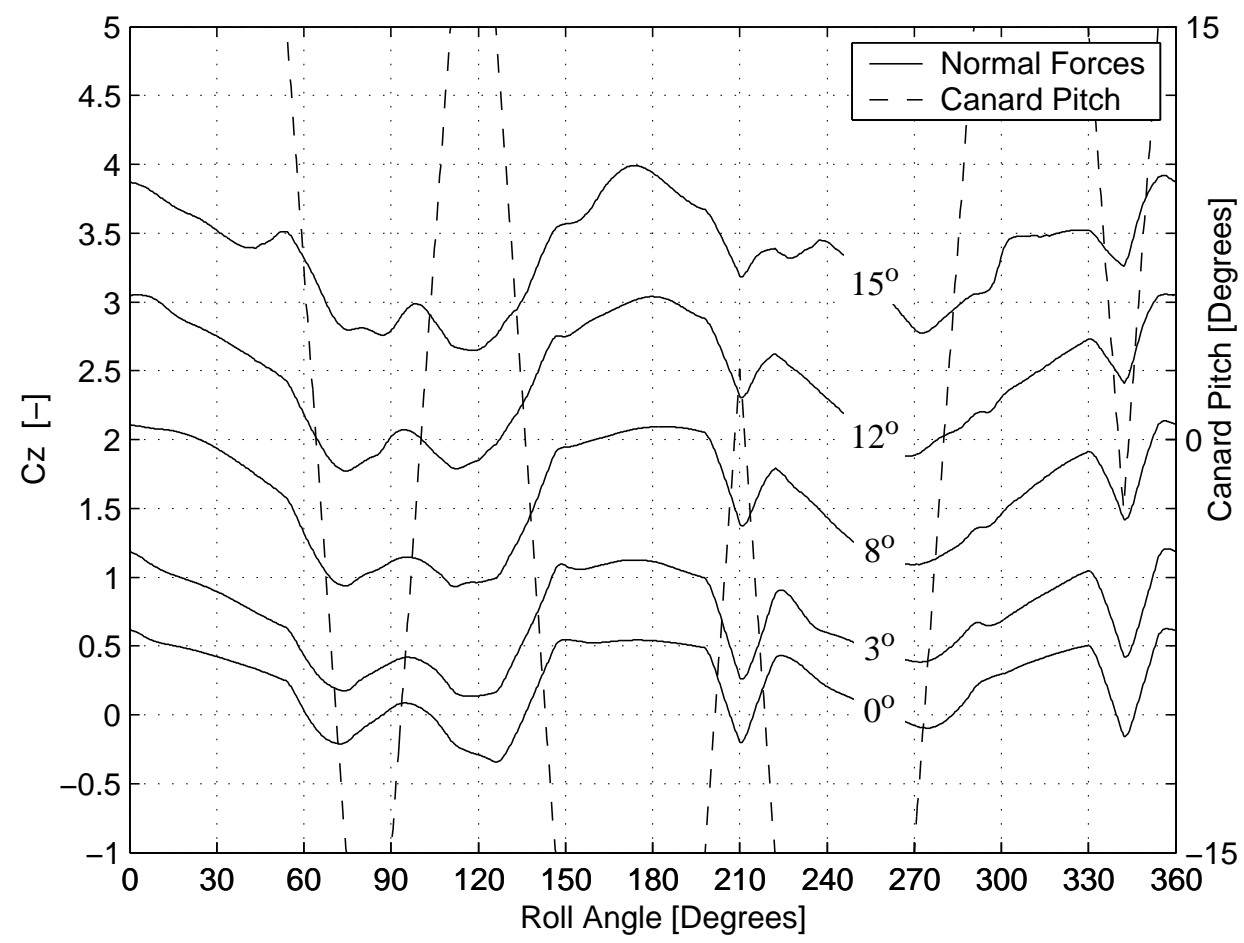

Figure 33. NORMAL FORCE coefficient for different $\alpha$ at $100 \%$ command level.

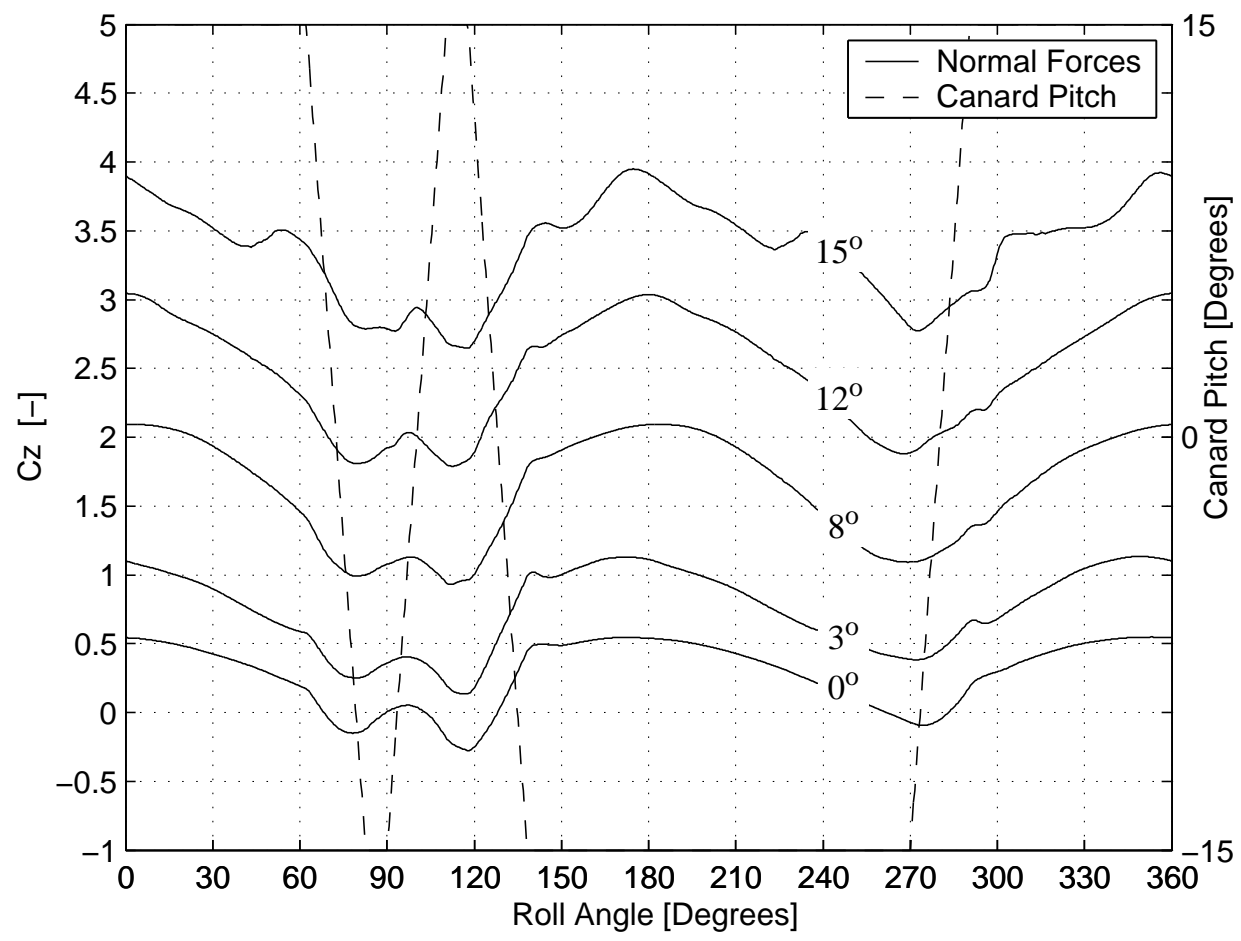

Figure 34. NORMAL FORCE coefficient for different $\alpha$ at 200\% command level. 
the $2^{\mathrm{o}}$ and $4^{\mathrm{O}}$ angle-of-attack fall smoothly between neighboring data points, and are omitted in the figures for clarity. The effect of increased $\alpha$ at each command level is a shift of the normal force curves upwards, with a limited change in variation with roll-angle. The direct contribution of the canards to instantaneous normal force dominate all other sources. However, the fuselage and tail section contributions are not insignificant (recall Figures 20 through 22). Still, the variation in fuselage and tail-section contributions to normal force are a secondary effect of canard position and resulting vortex/ body interaction.

The side forces for the 7 cases at $0 \%$ command level are shown in Figure 35. Again, results for $\alpha=2^{\circ}$ and $4^{\circ}$ are omitted for clarity. The effect of $\alpha$ here is less clear than for the normal force. An increase of $\alpha$ increases the variation of side force. An interesting result apparent in Figure 35 are non-zero side forces at roll-angles of $0^{\circ}$ and $180^{\circ}$ (canards horizontal). This is due to the induced circulation from the missile spin interacting with the vertical component of the upstream velocity vector. This is similar to the Magnus effect on a spinning golf-ball.

The roll-averaged normal forces are shown in Figure 36 and, consistent with the trend in Figures 32 through 34, increase with command level. The rollaveraged side forces are shown in Figure 37. The sideforces increase with command level. Further, at each command level, the side force goes from positive to negative with increasing angle-of-attack. The negative side-force at high $\alpha$ is caused partly by the Magnus effect as mentioned earlier. For a rotating cylinder, the Kutta-Joukowski theorem can be evaluated as follows:

$C_{y}=-4 \sin \alpha \frac{\Omega L}{U_{\infty}}$

where $\alpha$ is the angle-of-attack, $\Omega$ the cylinder spin-rate, $L$ the cylinder length, and $U_{\infty}$ the speed of the freestream flow. The result is shown in Figure 37 for comparison.

The roll-averaged pitching moments about the center of gravity are shown in Figure 38. The increase in pitching moment with command level is consistent with the increase in normal force for the canard. A change in command level to maneuver the missile affects the pitching moment in a similar manner for different $\alpha$.

The roll-averaged yawing moments about the center of gravity are shown in Figure 39. The negative contribution from an increase of command level is consistent with the increase in canard side-force. The effect of

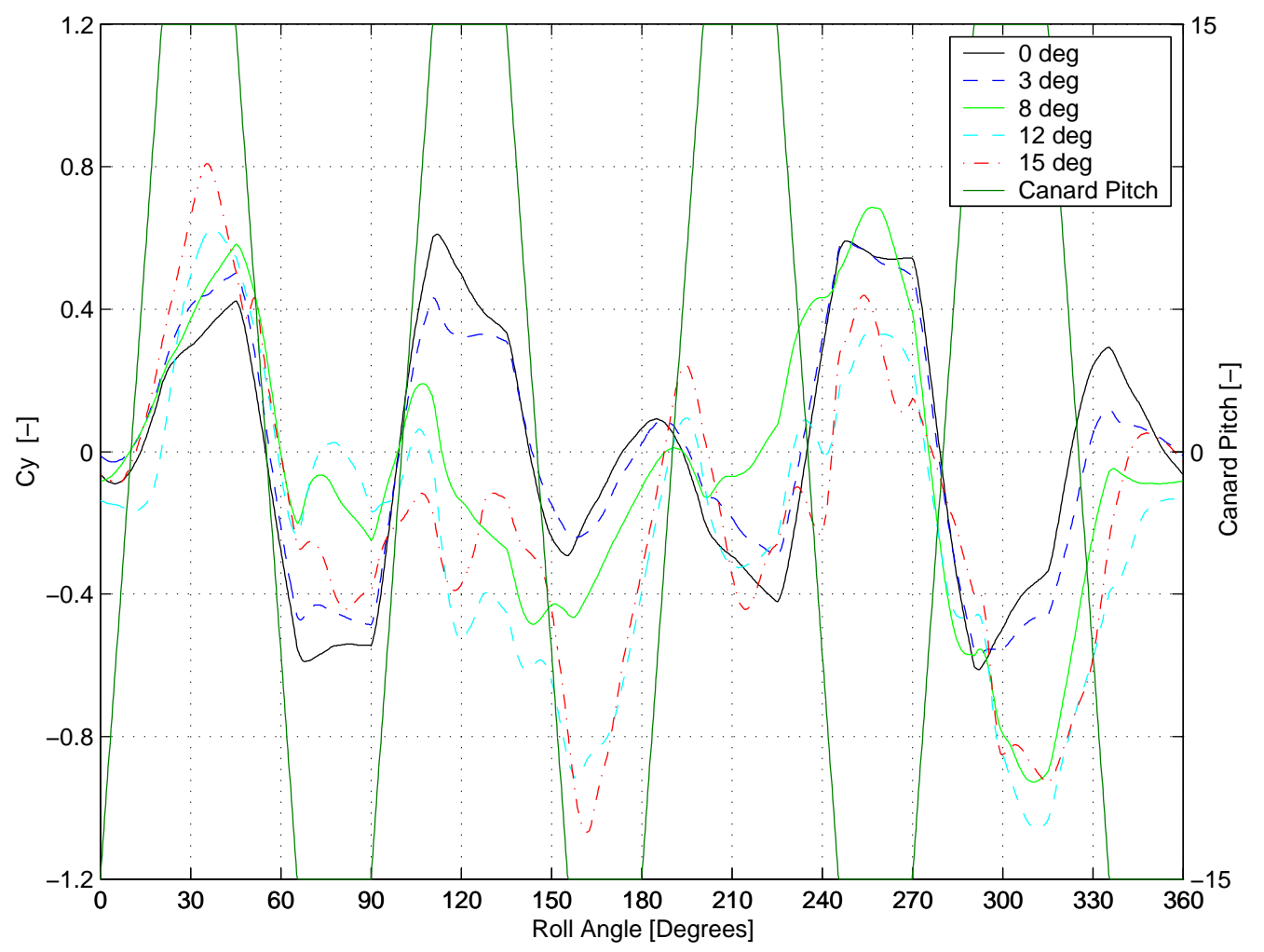

Figure 35. SIDE FORCE coefficient for different $\alpha$ at $0 \%$ command level. 


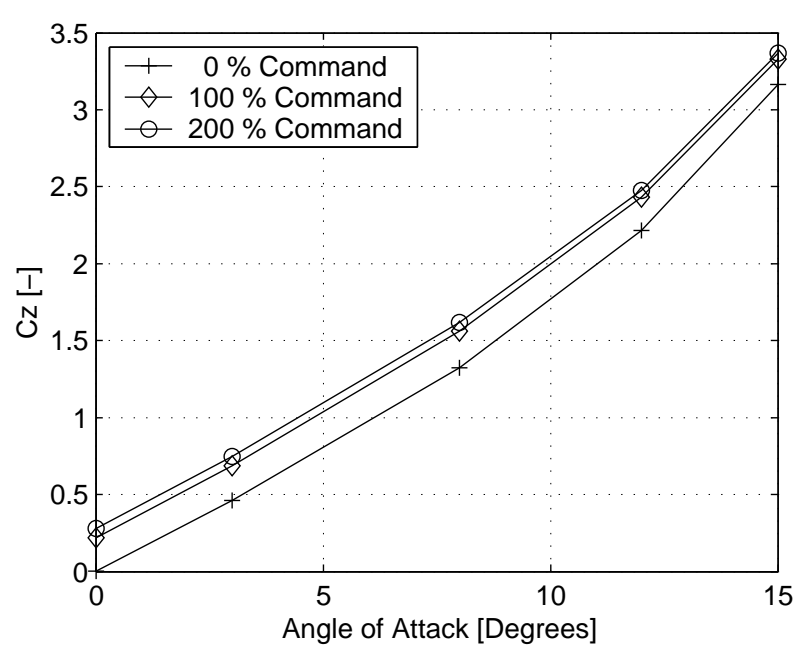

Figure 36. Roll-Averaged NORMAL FORCE coefficients for the Case Matrix.

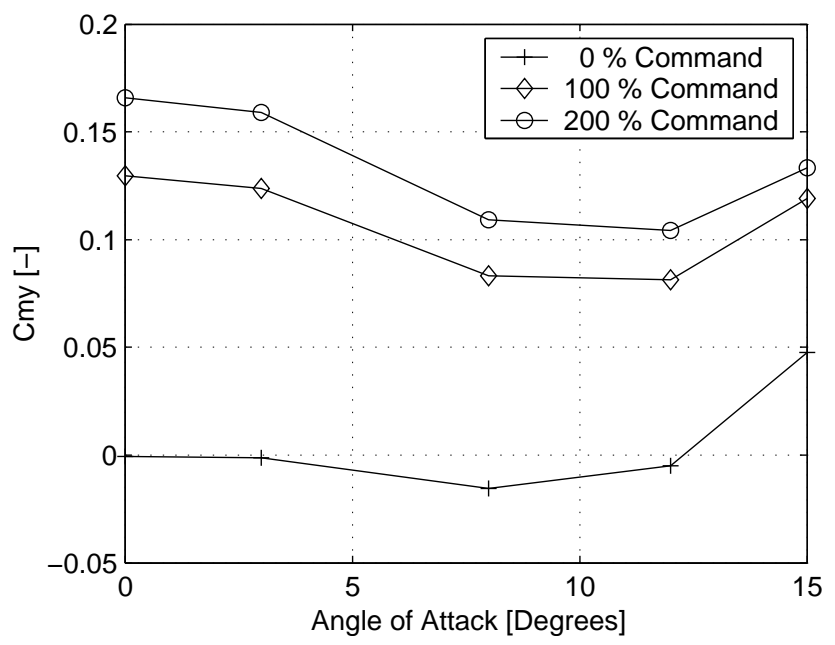

Figure 38. Roll-Averaged PITCHING MOMENT coefficients for the Case Matrix.

induced circulation seen on the side-force hardly modifies the yawing moment about the center of gravity, since it is relatively evenly distributed along the missile length.

\subsection{Interpolation of Results}

The roll-averaged inertial-frame forces and moments are the object of analysis in preceding sections of this paper. Indeed, the unsteadiness seen in instantaneous results is primarily caused by motion of the canards. However, comparison between canard motion and resulting forces and moments is complicated by the rotating framework used for presentation of the pitching sequence. It is not obvious, for example, that the computed force in Figure 30 corresponds to the pitching

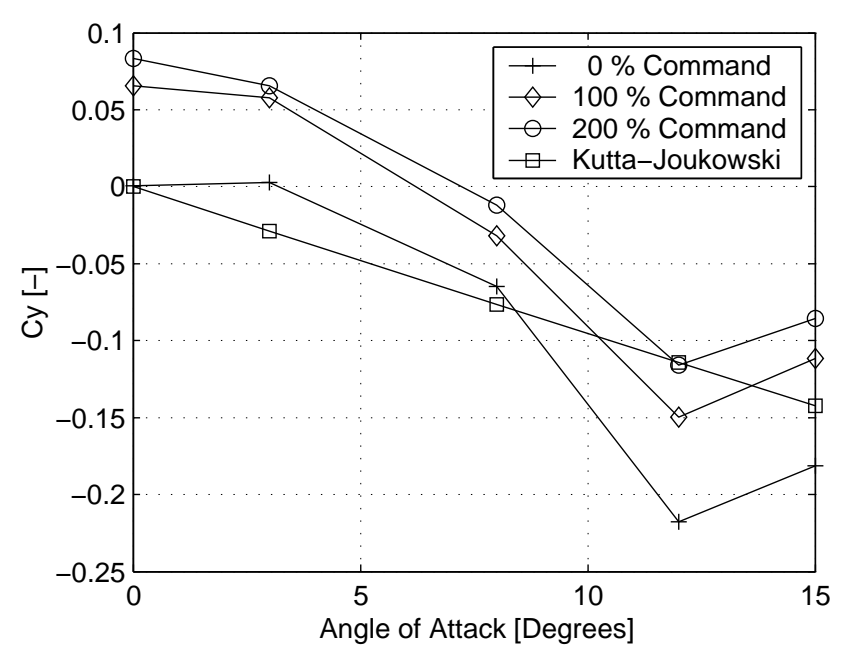

Figure 37. Roll-Averaged SIDE FORCE coefficients for the Case Matrix.

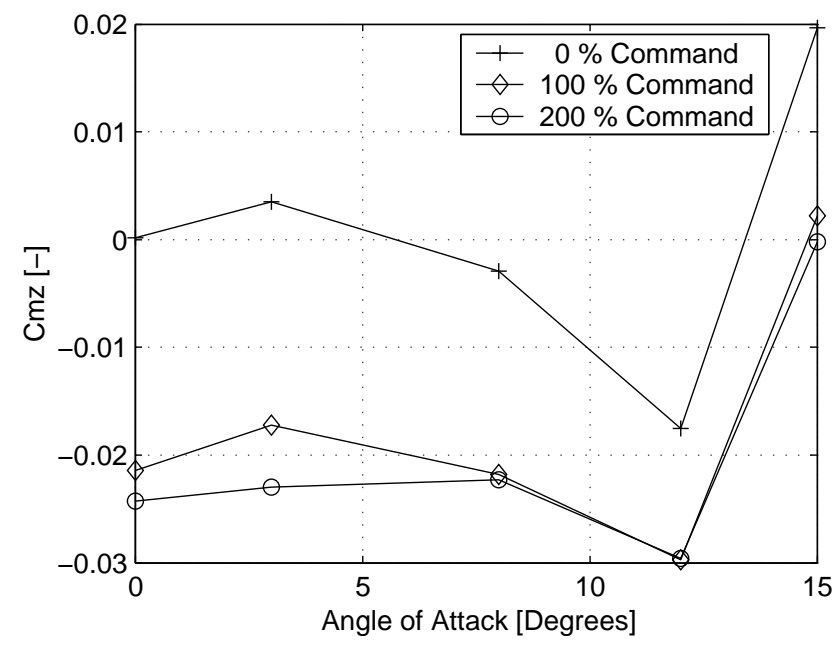

Figure 39. Roll-Averaged YAWING MOMENT coefficients for the Case Matrix.

sequence in the same figure. Looking at the three pitching sequences in Figure 31, it is not immediately clear what to expect of changes in roll-averaged forces when the command level is changed, and if these changes can be modeled well by linear interpolation, for example.

A method of classifying a canard pitching sequence is proposed here to aid in the understanding of computed results, and to provide a tool for interpolation of roll-averaged forces and moments between different command levels. The approach taken is to derive a measure of the aerodynamic force on the canards as a function of canard orientation, which is basically the same approach as using angle-of-attack to evaluate lift on a 2D airfoil. Here, the force (vector) is averaged over one missile revolution to represent the mean force vector on 
the canards. As discussed earlier, the canard forces are not the only contributors to changes in overall missile forces, but should be the best single-parameter approximation.

Two cartesian right-handed co-ordinate systems are used in the analysis. The $x-y-z$ system is in the inertial frame, with the $\mathrm{x}$-axis pointing nose to tail, $\mathrm{y}$-axis pointing to the side, and the $\mathrm{z}$-axis pointing up (see Figure 40). The $r-s-t$ system is initially oriented as the $x-y-z$ system, corresponding to horizontal canards with zero pitch. However, the $r-s-t$ system rotates with the canards. The movement of the $r-s-t$ basis vectors are evaluated as follows:

$i$. The pitch axis $\vec{e}_{s}$ is rotated around the inertial frame $\mathrm{x}$-axis with the roll-angle.

ii. The $\vec{e}_{r}$ basis vector is rotated around the new $\overrightarrow{e_{s}}$ with the canard pitch angle.

iii. The $\vec{e}_{t}$ basis vector is computed as the crossproduct $\overrightarrow{e_{r}} \times \overrightarrow{e_{s}}$

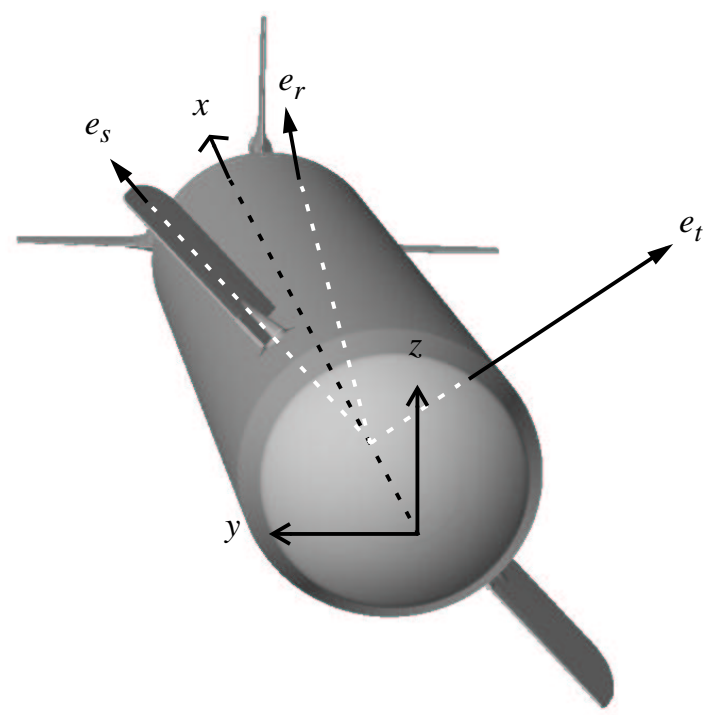

Figure 40. r-s-t rotating reference frame basis vecors relative to inertial frame $\mathrm{x}-\mathrm{y}-\mathrm{z}$ coordinate axes.

The canard angle-of-attack vector, $\vec{\alpha}_{c}$, is proposed in Equation 2 as a single parameter approximation for changes in missile loads due to changes in canard command level.

$$
\begin{gathered}
\overrightarrow{\alpha_{c}}=\vec{e}_{t} \frac{u_{r}^{2}+u_{t}^{2}}{\vec{u} \cdot \vec{u}} \sin ^{-1}\left(\frac{u_{t}}{\sqrt{u_{r}^{2}+u_{t}^{2}}}\right) \\
\text { (1) (2) }
\end{gathered}
$$

$\vec{u}$ is the upstream velocity vector at the missile angle-ofattack, $\alpha$. It has components $u_{r}, u_{s}$, and $u_{t}$ in the rotating system.

The three terms in Equation 2 are:

1 A direction vector normal to the canard. This approximates the direction of the force acting on the canard.

2 A fraction of the dynamic pressure, varying between 0 and 1 . This reduces the force on the canard according to the local "side-slip" angle.

3 The local angle-of-attack in the $r$-s- $t$ system

$\vec{\alpha}_{c}$ is a measure of the force acting on the canard, expressed as an angle-of-attack. The inertial frame components of this vector are defined as axial, side, and normal canard angles-of-attack (viz., $\alpha_{\mathrm{cx}}, \alpha_{\mathrm{cy}}$, and $\alpha_{\mathrm{cz}}$ ).

The "effective" canard angle-of-attack vector, $\overrightarrow{\alpha_{c e}}$, is obtained by averaging $\overrightarrow{\alpha_{c}}$ through one period of the pitching sequence. The inertial frame components of this quantity are intended to describe the effect of a canard pitching sequence on the roll-averaged forces. Figure 41 shows results for a case for $\alpha=0^{\circ}$ and command level $100 \%$. The solid line is the $\mathrm{C}_{\mathrm{z}}$ obtained from the V2 simulation results (scale shown on the left vertical axis). The dashed line is the canard normal angleof-attack, $\alpha_{c z}$, computed from the pitching sequence (scale shown on the right vertical axis). The dotted line is the canard pitching sequence (scale shown on the right vertical axis). The scales were chosen to show the excellent correlation between the V2 simulation results and the geometric quantity $\alpha_{c z}$ (canard normal angleof-attack). The side-force shows similar behavior. This indicates that the canard angle-of-attack vector is a useful estimator of forces on the canards.

The effective canard angles-of-attack (i.e., rollaveraged inertial frame components of $\vec{\alpha}_{c}$ ) for the three canard pitching sequences at $\alpha=0^{\circ}$ are listed in Table 4. These numbers are computed from the pitching sequence alone, and can be used as a first guess for trends in roll-averaged forces. An increase in command level gives mainly an increase in the effective normal canard angle-of-attack $\left(\alpha_{c e z}\right)$, but also gives small increases in $\alpha_{c e y}$ and $\alpha_{c e x}$. Increasing the command level from $100 \%$ to $200 \%$ increases the effective normal canard angle-of-attack by $27 \%$. The corresponding increase for the computed normal force for the V2 solution is also $27 \%$. The corresponding numbers for effective side canard angle-of-attack and V2 solution side force show increases of $19 \%$ and $26 \%$, respectively. These are also in reasonably good agreement considering that the side forces are small. 


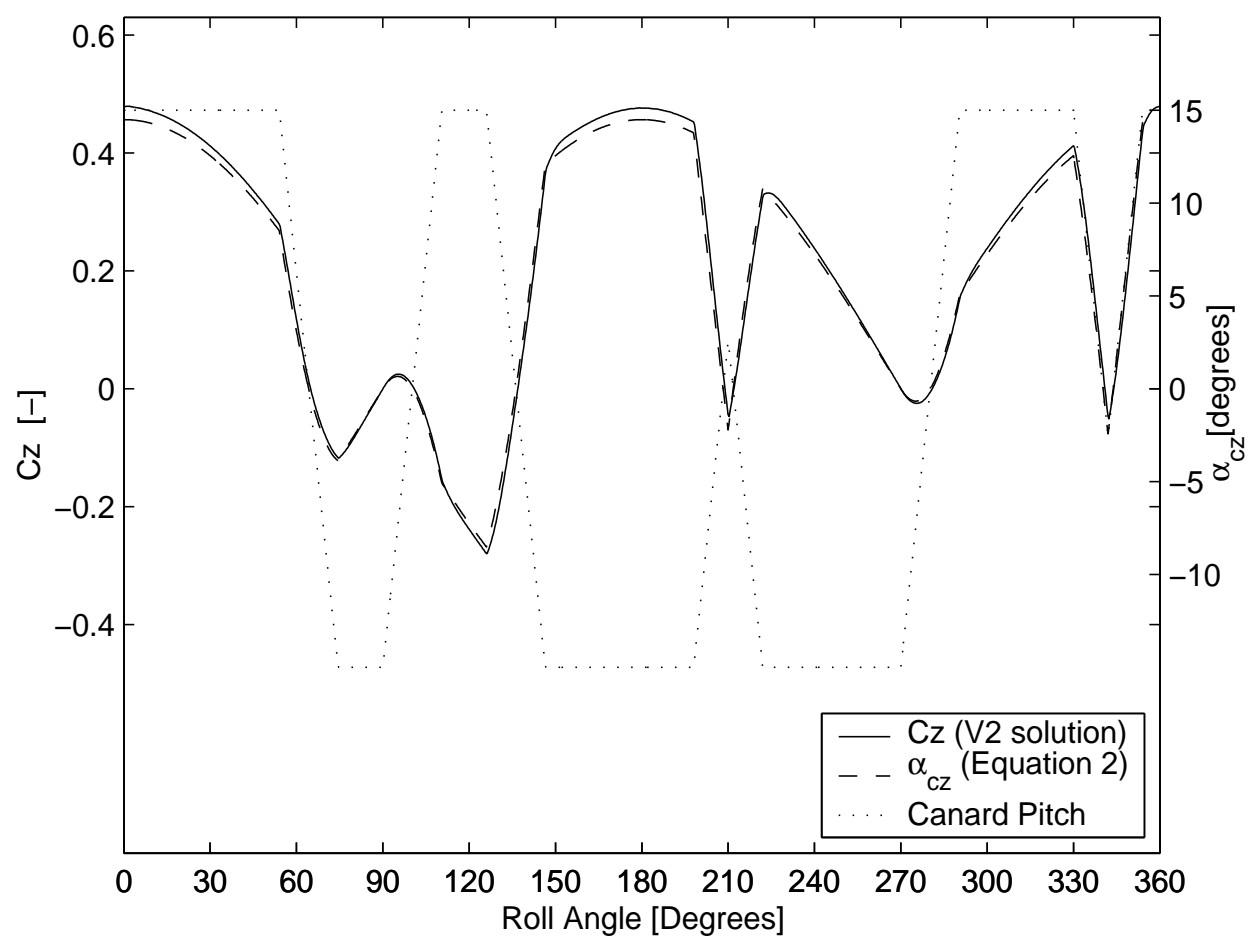

Figure 41. Canard NORMAL FORCE coefficient and angle-of-attack, $\alpha=0^{\circ}, \boldsymbol{c}=100 \%$

Table 4. Effective Canard Angles-of-attack ${ }^{*}$

\begin{tabular}{|c|ccc|}
\hline \multirow{2}{*|}{$\alpha_{c}$} & \multicolumn{3}{|c|}{ Command level } \\
& $0 \%$ & $100 \%$ & $200 \%$ \\
\hline$\alpha_{\text {cex }}$ (axial) & $2.7^{\mathrm{O}}$ & $2.9^{\mathrm{O}}$ & $3.3^{\circ}$ \\
$\alpha_{\text {cey }}$ (side) & $0^{\mathrm{o}}$ & $1.2^{\mathrm{o}}$ & $1.4^{\mathrm{o}}$ \\
$\alpha_{\text {cez }}$ (normal) & $0^{\mathrm{o}}$ & $6.3^{\mathrm{o}}$ & $8.0^{\mathrm{O}}$ \\
\hline
\end{tabular}

* Effective angles for three pitching sequences at free-stream angle-of-attack, $\alpha=0^{\circ}$.

In other words, lacking simulation (or experimental) data for $200 \%$ command level, good estimates of the same are easily obtainable based on linear extrapolation as a function of effective canard angles-of-attack. Linear extrapolation based on command level would fail, indicating an increase of $100 \%$ for both side force and normal forces. This illustrates that the roll-averaged forces are non-linear functions of the command level, and that effective canard angles-of-attack are better suited to aid interpolation and extrapolation of results between command levels.

\section{CONCLUSIONS}

A generic missile has been defined and analyzed to demonstrate the advantages and limitations of an overset grid approach to the aerodynamic performance prediction of rolling airframe missile systems. A computational investigation has been carried out to determine the resolution required for accurate prediction of surface loads and to determine the relative aerodynamic significance of viscous and missile roll-rate effects. The aerodynamic performance of the missile as a function of angle-of-attack and canard pitching sequence has also been considered.

A moderate viscous grid system of 8 million points is a good compromise between solution accuracy and case throughput. Instantaneous and roll-average forces and moments from the moderate viscous grid are comparable to those obtained in the high resolution baseline solution. Grid convergence of all forces and moments (except drag and rolling moment) were obtained.

Significant viscous effects are apparent for the rolling airframe missile system considered. An inviscid solution has no capacity to account for interactions between the canard vortices and the missile boundary layer. The consequence of this inadequacy is incorrect positioning and strength of the inboard canard vortices and incorrect prediction of the secondary effects of the canards on fuselage and fin surface pressures. The inviscid results match well with the viscous solutions when the canards are in transition between maximum positive and negative deflection angles. During this 
interval, the canard vortices are of minimal strength and the inviscid flow approximation is good.

Variation of roll-rate in the range of 0 to $10 \mathrm{~Hz}$ does not have a significant affect on roll-averaged forces and moments for the FM-3 missile. The effects on instantaneous loads are also minimal. The effects manifest as small roll-angle leads or lags in the load history.

A single parameter method of approximation for changes in missile loads due to changes in canard pitch angle command level is presented. The approximation method helps interpret the physical significance of simulation results and provides a powerful tool for interpolation of results between known data points. As such, the method is a quick and inexpensive means of supplying missing data, or for database expansion.

An overset structured grid domain decomposition method enables accurate and efficient simulation of rolling airframe missile configurations that involve relative motion between system components. Case throughput rates are sufficient to contemplate aerodynamic database population with Navier-Stokes solutions. It is significant to note that time-accurate simulation for one rolling airframe case with OVERFLOW-D is much less expensive than generating the same data via corresponding quasi-static solutions for needed roll-angles and canard pitch positions. Each quasi-static solution costs a significant fraction of a single time-accurate rolling airframe solution. A combination of time-accurate simulations for a relatively small number of pitching sequences and the canard angle-of-attack based approximation method represent a powerful way to quickly populate a large aerodynamic data base.

\section{ACKNOWLEDGEMENTS}

The authors gratefully acknowledge the contributions of Mr. Michael Aftosmis and Dr. Scott Murman of NASA Ames Research Center. They provided help in converting the FM-3 missile geometry definition into a useful format and participated with the authors in many helpful and interesting discussions. The expert consultation and assistance provided by Mr. Mark Potsdam of the U.S. Army Aeroflightdynamics Directorate at NASA Ames Research Center is also gratefully acknowledged. This work was supported in part by a grant of computer time from the DoD High Performance Computing Modernization Program at the ARL Major Shared Resource Center.

\section{$\underline{\text { REFERENCES }}$}

[1] Steger, J., Dougherty, C., and Benek, J., "A Chimera Grid Scheme," Advances in Grid Generation, K. N. Ghia and U. Ghia, eds., ASME FED-Vol 5., June 1983.

[2] Meakin, R., "Automatic Off-Body Grid Generation for Domains of Arbitrary Size," AIAA-2001-2536, 15th AIAA Computational Fluid Dynamics Conf., June 2001, Anaheim, CA.

[3] Meakin, R., "Adaptive Spatial Partitioning and Refinement for Overset Structured Grids" Comput. Methods Appl. Mech. Engrg., Vol. 189 (2000), 1077-1117.

[4] Chan, W., "The OVERGRID Interface for Computational Simulations on Overset Grids" AIAA Paper 2002-3188, 32nd AIAA Fluid Dynamics Conference, St. Louis, Missouri, June, 2002.

[5] Chan, W., Meakin, R., and Potsdam, M., "CHSSI Software for Geometrically Complex Unsteady Aerodynamic Applications," AIAA 2001-0539, AIAA 39th Aerosciences Meeting and Exhibit, January, 2001, Reno, NV.

[6] Buning, P. G., Jespersen, D. C., Pulliam, T. .H, Chan, W. M., Slotnick, J. P., Krist, S. E. and Renze, K. J., "OVERFLOW User's Manual," Version 1.8, NASA Langley Research Center, 1998. 